SLAC-PUB-95-6772

GPP-UdeM-TH-95-17

TRI-PP-95-05

\title{
NEW PARTICLES AND INTERACTIONS
}

\author{
A. Djouadi ${ }^{a}$ J. $^{N^{b}}{ }^{b}$ and T. G. Rizzo \\ ${ }^{a}$ Groupe de Physique des Particules, Université de Montréal, \\ Montréal $P Q, H 3 C$ 3J7 Canada. \\ ${ }^{b}$ TRIUMF, Vancouver BC, V6T 2A3 Canada. \\ ${ }^{c}$ Stanford Linear Accelerator Center, Stanford University, \\ Stanford, CA 94309 USA.
}

Contributors: D. Atwood, G. Azuelos, G. Bélanger, G. Bhattacharya, J. Blümlein, P. Chiappetta, M. Doncheski, R. Garisto, S. Godfrey, C. Greub, H.E. Haber, C. Heusch, J.L. Hewett, R. Harris, K. Huiti, A. Kagan, P.A. Kalyniak, J.L. Kneur, J. Layssac, N. Leporé, D. London, J. Maalampi, P. Minkowski, H. Nadeau, E. Nardi, D. Ng, S. Pakvasa, K.A. Peterson, A. Pomarol, M. Raidal, F.M. Renard, E. Roulet, M. Sher, J. Soares, M. Spira, B. Thorndyke, D. Tommasini, C. Verzegnassi.

\begin{abstract}
We analyze the manifestations of new matter particles predicted by models of new physics beyond the Standard Model, at present and future high-energy colliders. We consider both the production of these new particles and some of their indirect signatures at $p p$ and $e P$ colliders as well as $\mathrm{TeV} e^{+} e^{-}$colliders with their $e^{+} e^{-}, e \gamma, \gamma \gamma$ and $e^{-} e^{-}$modes. The report is arranged into four main sections plus an overview. These sections will deal separately with exotic and excited fermions, difermions, and new interactions.
\end{abstract}

To appear as a chapter in Electroweak Symmetry Breaking and Beyond the Standard Model, edited by T. Barklow, S. Dawson, H.E. Haber and S. Siegrist, World Scientific.

\footnotetext{
${ }^{1}$ Work supported by the Department of Energy, contract DE-AC03-76SF00515.
} 


\section{Overview}

Many theories beyond the Standard Model (SM) of the electroweak and strong interactions, predict the existence of new matter particles. These new particles can be cast into three categories: exotic fermions, excited fermions and difermions. A fourth category will consist in supersymmetric particles but these will be discussed in a different report.

a) Exotic Fermions. New fermions are predicted by many gauge extensions of the SM and often, they have the usual lepton and baryon quantum number but non-canonical SU $(2)_{L} \times \mathrm{U}(1)_{Y}$ quantum numbers, e.g. the left-handed (LH) components are in weak isosinglets and/or the right-handed $(\mathrm{RH})$ components in weak isodoublets. Examples of these exotic fermions are the following 11 .

i) Sequential fermions: they exist in the simplest extension of the SM where one simply has to add to the known fermionic spectrum with its three-fold replica a fourth family with the same quantum numbers. The existence of a fourth generation is still allowed by experimental data, if the associated neutrino is heavy enough [2]. This heavy neutrino should have a RH component in order that one can generate its mass, using the Higgs mechanism, in a gauge invariant way.

ii) Vector fermions: these occur for instance in the $\mathrm{E}_{6}$ group [3], which is suggested as a low energy limit of superstring theories. In $\mathrm{E}_{6}$, each fermion generation lies in the representation of dimension $\mathbf{2 7}$, and in addition to the fifteen SM chiral fields, twelve new fields are needed to complete this representation. Among these, there will be two weak isodoublets of heavy leptons, a RH and a LH one.

iii) Mirror fermions: they have chiral properties which are opposite to those of ordinary fermions, i.e. the $\mathrm{RH}$ components are in weak isodoublets and the LH ones are in weak isosinglets; there is also a LH heavy neutrino [4]. These fermions appear in many extensions of the SM and provide a possible way to restore leftright symmetry at the scale of the electroweak symmetry breaking; they naturally occur in lattice gauge theories [0].

$i$ v) Singlet fermions: these are the most discussed fermions in the literature, a prominent example being the $\mathrm{SO}(10)$ neutrino [6, 7, 8]. Indeed, in this unifying group, which is one of the simplest and most economic extensions of the SM, the smallest anomaly-free fermion representation has dimension 16. It contains a $\mathrm{RH}$ neutrino in addition to the 15 Weyl fermions in one fermion generation; this neutrino is of the Majorana type. Singlet neutrinos, which can be either of Majorana or Dirac type, and new singlet quarks also occur in $\mathrm{E}_{6}$ [3].

It is conceivable that these fermions, if for instance they are protected by some symmetry, acquire masses not much larger than the Fermi scale. This is very likely and even necessary, if the new gauge bosons which are generic predictions of the unified theories are relatively light [9]. In the case of sequential and mirror fermions (at least in the simplest versions of the models where the symmetry and the symmetry breaking pattern is the same as in the SM), theoretical arguments 
based on the unitarity of scattering amplitudes suggest that the masses should not exceed a few hundred $\mathrm{GeV}$ [10]. These particles could be therefore accessible at the next generation of colliders [1, 17, 8, 11, 12].

b) Excited Fermions. The existence of excited particles is a characteristic signal of substructure in the fermionic sector [13]. Indeed, if the known fermions are composite, they should be regarded as the ground state to a rich spectrum of excited states; the latter tumble down via a magnetic type de-excitation to the fundamental particles. In analogy with systems of substructure spanning from molecular to atomic then hadronic classifications, one hopes to explain in this way the well-ordered pattern of the fermionic spectrum with its three-fold replica, although there is not yet a satisfactory and predictive dynamical model.

In the simplest phenomenological models, excited fermions are assumed to have spin and isospin $1 / 2$, and that both their $\mathrm{LH}$ and $\mathrm{RH}$ components are in weak isodoublets so that they acquire their masses prior to $\mathrm{SU}(2)_{L} \times \mathrm{U}(1)_{Y}$ breaking. The transition between the excited and the fundamental states consisting of the ordinary $\mathrm{SM}$ particles can be described by an $\mathrm{SU}(3)_{C} \times \mathrm{SU}(2)_{L} \times \mathrm{U}(1)_{Y}$ invariant effective interaction of the magnetic type. Hence, the excited particles will have full couplings to the gauge bosons and therefore can be pair produced at colliders, and also magnetic-type couplings to ordinary fermions and gauge bosons (that are inversely proportional to the compositeness scale $\Lambda$ ) which will determine the decay of the excited states and allow for a new production mechanism: single production in association with their light partners; see Refs. [14, 15].

The search for excited fermions has been systematically pursued for more than thirty years without any sucess [16]. However, this situation is not in conflict with the motivation behind the introduction of excited particles: compositeness is often invoked as a possible alternative to the SM description of the electroweak symmetry breaking and it is conceivable that the first excitations from the new physics would only be felt at, or above, the Fermi scale. Therefore, future colliders operating at such energies will play an important role in testing this possibility.

c) Difermions. These are scalar or vector particles (spin $1 / 2$ difermions are also discussed in the context of supersymmetric theories) which have unusual baryon and/or lepton quantum numbers. Examples of these particles are as follows.

i) Leptoquarks (LQ): with $\mathrm{B}= \pm 1 / 3$ and $\mathrm{L}= \pm 1$ [17]. These particles are expected in Technicolor models in composite models (where quarks and leptons are made of the same subconstituents) as bound states of quark-lepton pairs and also in Grand Unified models (for instance in the $\mathrm{E}_{6}$ model, the supersymmetric partner of the exotic colored particle which lies in the $\mathbf{2 7}$ representation, can have leptoquark quantum numbers). The leptoquarks will have the usual gauge couplings to the photon, the $W / Z$ bosons and gluons (for spin-1 LQ's an anomalous magnetic moment can be added) and also Yukawa couplings to lepton-quark pairs which determine their decays. For not too heavy LQ's, this Yukawa coupling should be 
chiral in order to avoid that leptons acquire a too large magnetic moment 18.

A systematic description of leptoquarks quantum numbers and interactions can be made, starting from an effective Lagrangian with general dimensionless $\mathrm{SU}(3)_{C} \times \mathrm{SU}(2)_{L} \times \mathrm{U}(1)_{Y}$ invariant couplings and conserved lepton and baryon numbers [19]. With fermion number $F=3 B+L=0$ (the LQ couples to lepton-quark pairs) or 2 (the LQ couples to lepton-antiquark pairs), there are 10 leptoquarks: 5 scalars and 5 vectors (plus their charge conjugate states), with electric charges ranging from $1 / 3$ to $5 / 3$ in absolute value. The full set of these leptoquarks is present in a $\mathrm{SU}(15)$ based model of strong-electroweak unification [20].

ii) Diquarks: with $\mathrm{B}= \pm 2 / 3$ and $\mathrm{L}=0$. They are also predicted in composite models as bound states of quark pairs, and in Grand Unified models (in the model based on the $\mathrm{E}_{6}$ symmetry group, the supersymmetric partner of the exotic colored particle can also have diquark quantum numbers [3]).

iii) Dileptons with $\mathrm{B}=0$ and $\mathrm{L}= \pm 2$ [21]. These particles occur in theories where the electroweak gauge group for leptons is extended from $\mathrm{SU}(2)_{L} \times \mathrm{U}(1)_{Y}$ to $\mathrm{SU}(3)$ and baryon and lepton numbers are conserved. They can appear both as scalar and as vector gauge particles and can be singly or doubly charged; for instance, doubly charged dilepton gauge bosons appear in a SU(15) grand unification model. Dileptons have couplings to (ordinary) gauge bosons which are fixed by gauge invariance, and Yukawa couplings to leptons which mediate the decays.

All these difermions can have masses not too much larger than the electroweak symmetry breaking scale and therefore could be accessible at future colliders.

The presence of new physics beyond the Standard Model can manifest itself not only through the discovery of new particles but also, if the latter are too heavy to be directly produced, through new interactions which alter the SM predictions for conventional processes involving the known particles. One can then have an indirect evidence for new physics at a mass scale higher than the one being probed directly. In many cases, these new interactions can be expressed as higher dimensional, non-renormalizable, operators written in terms of the SM fields. In dealing with exotica, there is no limit to what new interactions may exist so any summary must necessarily be limited in scope.

One of the best known examples of this type is the possibility that the top quark may have anomalous interactions with the gauge bosons of the SM. Indeed, due to its large mass, the top quark may play a special role and may be the first place where non-standard effects will appear. These new interactions for top naturally divide themselves into those associated with QCD (i.e., modified $t \bar{t} g$ and $t \bar{t} g g$ vertices) and new electroweak couplings with $W, Z, \gamma$. In the QCD case, assuming $C P$ conservation, the lowest dimension operator representing new physics is the anomalous chromomagnetic moment, $\kappa$. A non-zero $\kappa$ at hadron colliders can lead to a significantly modified top pair production cross section with little effect on various distributions; the influence of $\kappa$ on single top production is quite small. At the NLC, this new coupling induces a high energy tail in the gluon energy 
distribution for the process $e^{+} e^{-} \rightarrow t \bar{t} g$. Both hadron and $e^{+} e^{-}$colliders can probe these new top quark QCD interactions. In the electroweak sector, one can look for the effects of a finite charge radius and magnetic dipole moment for the top quark in both $\gamma \gamma \rightarrow t \bar{t}$ or $e^{-} e^{+} \rightarrow t \bar{t}$ with very high sensitivity.

New four-point interactions between the SM fermions and gauge bosons can occur in a number of ways in addition to those required by gauge invariance. The simplest example is a dimension- $8 q \bar{q} \gamma \gamma$ operator which can lead to an excess of central diphoton pairs at large invariant mass at proton colliders. Searches for such interactions can probe compositeness scales of order several $\mathrm{TeV}$.

The existence of Technicolor-like vector particles that are strongly coupled to the SM gauge fields may also make their presence felt at scales below their direct production thresholds. Precision measurements at a $1 \mathrm{TeV} e^{+} e^{-}$linear collider can reveal the effects of such particles with masses in the few $\mathrm{TeV}$ region.

In this report we will analyze the manifestations of these new particles and interactions at future high-energy colliders. We will consider both the production of these new particles and some of their indirect signatures, at $p p$ colliders [LHC with $\sqrt{s}=14 \mathrm{TeV}], e P$ colliders $[\mathrm{LEP} \times \mathrm{LHC}$ with $\sqrt{s}=1.2 \mathrm{TeV}]$ and $e^{+} e^{-}$colliders [NLC with $\sqrt{s}=0.5-1 \mathrm{TeV}]$ with its $e^{+} e^{-}, e \gamma, \gamma \gamma$ and $e^{-} e^{-}$modes. The report is arranged into four main sections plus the Introduction. These sections will deal separately with exotic and excited fermions, difermions, and new interactions. 


\section{Exotic Fermions}

\subsection{Introduction}

Except for singlet neutrinos which have no electromagnetic and weak charges, the new fermions couple to the photon and/or to the electroweak gauge bosons $W / Z$ (and for heavy quarks, to gluons as well) with full strength. These couplings allow for the pair production of heavy leptons and quarks; in units of the proton charge, they are given by $\left(e^{F}\right.$ is the electric charge of the fermion $F, I_{3 L}^{F} / I_{3 R}^{F}$ the third components of $\mathrm{LH} / \mathrm{RH}$ isospin and $\left.s_{W}^{2}=1-c_{W}^{2} \equiv \sin ^{2} \theta_{W}\right)$

$$
v_{\gamma}^{F}=e^{F}, a_{\gamma}^{F}=0, v_{Z}^{F} \equiv v_{F}=\frac{2 I_{3 L}^{F}+2 I_{3 R}^{F}-4 e^{F} s_{W}^{2}}{4 s_{W} c_{W}}, a_{Z}^{F} \equiv a_{F}=\frac{2 I_{3 L}^{F}-2 I_{3 R}^{F}}{4 s_{W} c_{W}}
$$

If they have unconventional quantum numbers, the new fermions will mix with the SM fermions which have the same $\mathrm{U}(1)_{Q}$ and $\mathrm{SU}(3)_{C}$ assignments. This mixing will give rise to new currents which determine to a large extent their decay properties and allow for a new production mechanism: single production in association with their light partners. The mixing pattern depends sensitively on the considered model and, in general, is rather complicated especially if one includes the mixing between different generations. However, this inter-generational mixing should be very small since it would induce at the tree level, flavor changing neutral currents which are severely constrained by existing data [22].

In the present analysis, we will neglect the inter-generational mixing and treat the few remaining mixing angles as phenomenological parameters. To describe our parameterization, let us explicitly write down the interaction of the electron and its associated neutrino with exotic charged and neutral heavy leptons. Allowing for both LH and RH mixing, and assuming small angles so that one can write $\sin \zeta_{L, R} \simeq \zeta_{L, R}$, the Lagrangian describing the transitions between $e, \nu_{e}$ and the heavy leptons $N, E$ of the first generation is $\left(g_{W}=e / \sqrt{2} s_{W}\right.$ and $\left.g_{Z}=e / 2 s_{W} c_{W}\right)$

$$
\begin{aligned}
\mathcal{L} & =g_{W}\left[\zeta_{L}^{\nu E} \overline{\nu_{e}} \gamma_{\mu} E_{L}+\zeta_{R}^{\nu E} \bar{\nu}_{e} \gamma_{\mu} E_{R}\right] W^{\mu}+g_{Z}\left[\zeta_{L}^{e E} \bar{e} \gamma_{\mu} E_{L}+\zeta_{R}^{e E} \bar{e} \gamma_{\mu} E_{R}\right] Z^{\mu} \\
& +g_{W}\left[\zeta_{L}^{e N} \bar{e} \gamma_{\mu} N_{L}+\zeta_{R}^{e N} \bar{e} \gamma_{\mu} N_{R}\right] W^{\mu}+g_{Z}\left[\zeta_{L}^{\nu N} \overline{\nu_{e}} \gamma_{\mu} N_{L}+\zeta_{R}^{\nu N} \bar{\nu}_{e} \gamma_{\mu} N_{R}\right] Z^{\mu}+\text { h.c. }
\end{aligned}
$$

The generalization to the other lepton families and to quarks is obvious.

Let us now summarize the present experimental constraints on the masses of the new fermions and on their mixing with the ordinary ones. First, we will assume that the new gauge bosons predicted by the Grand Unified Models, will be too heavy 23] to affect the decays and the production of the exotic fermions. As previously discussed, we will only allow for a flavor-diagonal mixing; the latter will alter the couplings of the electroweak gauge bosons to light quarks and leptons from their SM values. Since these couplings have been very accurately determined at LEP1 (through the measurement of total and partial decay widths as well as forwardbackward and polarization asymmetries) and in various low-energy experiments 
and found to agree with the SM predictions up to the level of one percent, the mixing angles are constrained to be smaller than $\mathcal{O}\left(10^{-1}\right)$ 22]; these constraints are summarized in Table 1. In the case of leptons, if the $\mathrm{LH}$ and $\mathrm{RH}$ mixing angles have the same size, the precise measurement of $(\mathrm{g}-2)_{e, \mu}$ leads to even more stringent constraints, $\zeta<\mathcal{O}\left(10^{-2}\right)$ [24], so one has to set $\zeta_{L, R} \gg \zeta_{R, L}$.

Table 1: $90 \%$ C.L. upper limits on the ordinary-exotic flavor diagonal mixing angles for individual fits (one angle at a time is allowed to vary) and joint fits (all angles allowed to vary simultaneously). Only the results corresponding to neutrinos mixed with heavy singlet leptons are shown. In most of the cases, LEP measurements of partial widths and asymmetries give the most effective constraints. $s_{L}^{e}$ corresponds to $\sin \zeta_{L}^{e}$, etc..; $m_{t}=170 \mathrm{GeV}$ and $M_{H}=200 \mathrm{GeV}$ are assumed.

\begin{tabular}{|c|c|c|c|c|c|}
\hline & Individual & Joint & & Individual & Joint \\
\hline$\left(s_{L}^{e}\right)^{2}$ & 0.0016 & 0.0054 & $\left(s_{L}^{u}\right)^{2}$ & 0.0022 & 0.012 \\
$\left(s_{R}^{e}\right)^{2}$ & 0.0020 & 0.0018 & $\left(s_{R}^{u}\right)^{2}$ & 0.010 & 0.023 \\
$\left(s_{L}^{\mu}\right)^{2}$ & 0.0013 & 0.0049 & $\left(s_{L}^{d}\right)^{2}$ & 0.0026 & 0.016 \\
$\left(s_{R}^{\mu}\right)^{2}$ & 0.0019 & 0.0040 & $\left(s_{R}^{d}\right)^{2}$ & 0.0066 & 0.019 \\
$\left(s_{L}^{\tau}\right)^{2}$ & 0.0011 & 0.0037 & $\left(s_{L}^{s}\right)^{2}$ & 0.0036 & 0.019 \\
$\left(s_{R}^{\tau}\right)^{2}$ & 0.0018 & 0.0034 & $\left(s_{R}^{s}\right)^{2}$ & 0.021 & 0.059 \\
$\left(s_{L}^{\nu_{e}}\right)^{2}$ & 0.0053 & 0.0053 & $\left(s_{L}^{c}\right)^{2}$ & 0.0044 & 0.024 \\
$\left(s_{L}^{\nu_{\mu}}\right)^{2}$ & 0.0020 & 0.0052 & $\left(s_{L}^{b}\right)^{2}$ & 0.0097 & 0.043 \\
$\left(s_{L}^{\nu_{\tau}}\right)^{2}$ & 0.0055 & 0.017 & $\left(s_{R}^{b}\right)^{2}$ & 0.0017 & 0.031 \\
\hline
\end{tabular}

From the negative search of new states and from the measurement of $Z$ decay widths at LEP1, one can infer a bound of the order of $M_{Z} / 2$ on the masses of the new fermions [2] independently of their mixing, except for singlet neutrinos. Masses up to $m_{F} \sim M_{W}$ can be probed at LEP2. In the case of heavy neutrinos, including the gauge singlets, an additional constraint is provided by the negative search 25] of these states through single production in $Z$ decays: if the $\nu N$ mixing angle is of the order of $\sim 0.1$ or larger, $m_{N}$ should be larger than $M_{W}$ [25]; a similar mass bound can be established for the heavy charged lepton. Note that for mixing angles much smaller than $\mathcal{O}\left(10^{-2}\right)$, no bound can be derived on the singlet neutrinos masses: the production cross section is small and/or the heavy neutrino escapes detection because of its too long decay length.

The heavy fermions decay through mixing into massive gauge bosons plus their ordinary light partners; for masses larger than $M_{W} / M_{Z}$ the vector bosons will be on-shell. Using the scaled masses $a_{V}=M_{W, Z}^{2} / m_{F}^{2}$, the decay widths are 12]

$$
\Gamma\left(F_{L, R} \rightarrow V f\right)=\frac{\alpha \delta_{V}}{32 s_{W}^{2} c_{W}^{2}}\left(\zeta_{L, R}^{f F}\right)^{2} \frac{m_{F}^{3}}{M_{Z}^{2}}\left(1-a_{V}\right)^{2}\left(1+2 a_{V}\right)
$$


with $\delta_{V}=1(2)$ for $Z(W)$. For small mixing angles, the heavy fermions have very narrow widths: for $\zeta_{L} / \zeta_{R} \sim 0.1$ and masses around $100 \mathrm{GeV}$ the partial decay widths are less than $10 \mathrm{MeV}$. The decay widths increase rapidly for increasing fermion masses, $\Gamma \sim m_{F}^{3}$, but for allowed values of the mixing angles, they do not exceed the $10 \mathrm{GeV}$ range even for $m_{F} \sim \mathcal{O}(1 \mathrm{TeV})$. The charged current decay mode is always dominant and for fermion masses much larger than $M_{Z}$, the branching ratios are $1 / 3$ and $2 / 3$ for the neutral and charged current decays, respectively. For Majorana neutrinos []], both the $l^{-} W^{+} / \nu_{l} Z$ and $l^{+} W^{-} / \overline{\nu_{l}} Z$ are possible; this makes its total decay width twice as large as for Dirac neutrinos. To fully reconstruct the heavy leptons from their final decay products one needs the branching ratio of their decays into visible particles, for large $m_{L}$ they are given by ( $N$ is a Dirac neutrino)

$$
\begin{aligned}
\operatorname{Br}\left(E^{ \pm} \rightarrow Z l^{ \pm} \rightarrow j j l^{ \pm}\right) & \simeq 0.23 \\
\operatorname{Br}\left(N \rightarrow W^{+} l^{-} \rightarrow j j l^{-}\right) & \simeq 0.43
\end{aligned}
$$

In the case of $E$, one can also include the cleaner $Z \rightarrow e^{+} e^{-}+\mu^{+} \mu^{-}$decays, but the branching ratio is rather small: $\sim 6 \%$ compared to $\sim 70 \%$ for $Z \rightarrow$ hadrons. Finally, we note that cascade decays are also possible: either two leptons are in the same isodoublet and the heaviest one can decay into the lighter, or the mixing between heavy leptons is much larger that the heavy-light lepton mixing in which case, the decay of a heavy lepton into a lighter one first will be favored; these cascade decays will not be considered here; for some details see ref. [12.

A possibility that should not be overlooked is that a heavy charged lepton could be quite long-lived, with a lifetime long enough to leave a visible track in a detector [26] (for long-lived quarks, see e.g. Ref. [27]). A simple model with such a lepton would be a model in which the heavy leptons form a vectorlike doublet, and in which mixing with the lighter generations is either absent or suppressed by ratios of neutrino masses (which then would give a mixing angle less than $10^{-10}$ ). In this case, the charged lepton and neutrino are degenerate in mass at tree level. Radiative corrections will break this degeneracy, and will give a mass splitting of between 270 and $330 \mathrm{MeV}$ (as the lepton mass ranges from $100 \mathrm{GeV}$ to $800 \mathrm{GeV}$ ) ; this remarkable insensitivity to the lepton mass is reflected in the lepton lifetime, which ranges from 1.2 to 2.0 nanoseconds. Such a particle would leave a visible track, then decay into neutrals plus a low energy electron or muon pair. With such a clear signature, the discovery reach at a hadron collider would be much higher than for conventional heavy leptons, and would be at the kinematic limit for $e^{+} e^{-}$ colliders. It turns out that if one assumes that this doublet lies in a supersymmetric theory, then the resulting lifetime is virtually independent of the supersymmetry parameter space, and is unchanged from the non-supersymmetric case.

Such a model is not particularly unusual [28]. For example, the leptonic extension of Frampton and Kephart aspon model (which offers a solution to the strong $\mathrm{CP}$ problem) has such a doublet with small mixing. In the model of Griest and 
Sher, extra generations of Higgs doublets in SUSY were considered. Assuming only that a symmetry suppresses FCNC at tree level, it has been shown that the additional Higgsinos form a doublet like the above model. The extra Higgs bosons themselves have the unusual property that the second lightest (neutral) is a few hundred $\mathrm{MeV}$ heavier than the lightest (neutral), leading to a low energy electron or muon pair coming from a point detached from the interaction; here backgrounds may be considerable, and are under investigation [26].

Constraints on a fourth generation SM quarks $\left(t^{\prime}, b^{\prime}\right)$ and leptons $(N, E)$ requires a special discussion [29]. We assume that $m_{t^{\prime}}>m_{t}$ and that there is no Majorana mass for the RH neutrino. First, one needs to parameterize the CKM matrix for four generations; for a real $4 \times 4$ matrix, one has to introduce three new parameters $V_{u b^{\prime}} \equiv \epsilon, V_{c b^{\prime}} \equiv \delta$ and $V_{t b^{\prime}} \equiv \sin \theta$, and one has

$$
V \simeq\left(\begin{array}{cccc}
1 & \lambda & A \rho \lambda^{3} & \epsilon \\
-\lambda & 1 & A \lambda^{2} & \delta \\
A \lambda^{3}(1-\rho) \cos \theta-(\epsilon-\delta \lambda) \sin \theta & -A \lambda^{2} \cos \theta-\delta \sin \theta & \cos \theta & \sin \theta \\
-A \lambda^{3}(1-\rho) \sin \theta-(\epsilon-\delta \lambda) \cos \theta & A \lambda^{2} \sin \theta-\delta \cos \theta & -\sin \theta & \cos \theta
\end{array}\right)
$$

which preserves much of the Wolfenstein parameterization for the $3 \times 3$ case. The present experimental data constrain the Wolfenstein parameters to $\lambda=0.22, A=$ $0.79 \pm 0.12$ and $|\rho|=0.36 \pm 0.09$, and the upper bounds for the new parameters, obtained from unitarity, are given as $|\epsilon| \leq 0.077$ and $|\delta| \leq 0.594$. From the precision LEP data (mainly from the $\rho$ parameter and the ratio of the $Z \rightarrow b \bar{b}$ to the hadronic widths), one obtains for $m_{t}=150 \mathrm{GeV}$ and $M_{H}=300 \mathrm{GeV}, m_{b^{\prime}} \sim m_{t^{\prime}}$ for $\sin \theta \sim 0$ and $m_{b^{\prime}}>100 \mathrm{GeV}, m_{t^{\prime}}<300 \mathrm{GeV}$ for $\sin \theta \sim 1 / \sqrt{2}$. The LEP experiment tell us in addition that $m_{N}>45 \mathrm{GeV}$. CLEO's experimental bound on $b \rightarrow s \gamma$ as well as the differences $B_{d}-\bar{B}_{d}$ and $B_{s}-\bar{B}_{s}$ would constrain $\theta, m_{t^{\prime}}$ and also $\delta$ (for CLEO). The branching ratio for the decay $K^{+} \rightarrow \pi^{+} \nu \bar{\nu}$ would also place bounds on $\theta$ and $m_{t^{\prime}}$ as well as $\epsilon$ and $\delta ; D-\bar{D}$ mixing would give better constraint on the last two parameters. Finally, the heavy-light lepton mixing angles are constrained at the level of $\sim 0.1$ for the third generation (mainly from lepton universality) and at a level of $\sim 10^{-2}-10^{-3}$ for the two other generations (from FCNC processes like $\mu \rightarrow e \gamma, \mu \rightarrow 3 e$ and $\mu-e$ conversion in nuclei, etc..).

The fourth generation leptons $N$ and $E$ will decay into leptons and massive gauge bosons (the decay widths, up to mixing angles, are given in eq. 3). Because $\sin \theta$ is not necessary small, the decays of $t^{\prime}, b^{\prime}$ as well as $t$ depend on $m_{b^{\prime}}$. If $m_{t^{\prime}}<m_{b^{\prime}}, t^{\prime}$ will be produced first and will then mainly decay into $b+W$ or $s+W$ depending on the parameters $\sin \theta$ and $\delta$ (since the constraints on $\delta$ are not very stringent, $t^{\prime} \rightarrow s W$ is not necessary suppressed). In the opposite case, $m_{b^{\prime}}<m_{t^{\prime}}$, there are two possibilities: if $m_{b^{\prime}}>m_{t}+m_{W}$, the main decays will be $b^{\prime} \rightarrow t W$ and $b^{\prime} \rightarrow(c, u) W$ (if $\sin \theta>\delta, \epsilon$ the former mode is dominant, leading to a nice signature); if $m_{b^{\prime}}<m_{t}+m_{W}$, the FCNC decays $b^{\prime} \rightarrow b(g, \gamma, Z)$ can be important (if $\delta$ and $\epsilon$ are small, say less than $10^{-3}$ ) compared to the CC decays $b^{\prime} \rightarrow(c, u) W$, and $b^{\prime} \rightarrow b Z \rightarrow b l^{+} l^{-}$would provide a spectacular signature. 


\subsection{Production in $e^{+} e^{-}$Colliders}

If their masses are smaller than the beam energy, the new fermions can be pair produced in $e^{+} e^{-}$collisions: through $s$-channel $\gamma$ and $Z$ exchange for charged fermions, and only $Z$ exchange for heavy neutrinos. The differential cross section (with $\theta$ specifying the direction of the fermion $F$ with respect to the $e^{-}$) is [12]

$\frac{d \sigma}{d \cos \theta}=\frac{3}{8} \sigma_{0} N_{c} \beta_{F}\left[\left(1+\cos ^{2} \theta\right)\left(\sigma_{V V}+\beta_{F}^{2} \sigma_{A A}\right)+\left(1-\beta^{2}\right) \sin ^{2} \theta \sigma_{V V}+2 \beta_{F} \cos \theta \sigma_{V A}\right]$,

where $N_{c}$ is a color factor, $\sigma_{0}=4 \pi \alpha^{2} / 3 s$ the point-like QED cross section and $\beta_{F}=\left(1-4 m_{F}^{2} / s\right)^{1 / 2}$ is the velocity of the fermion in the final state; in terms of the $F F \gamma$ and FFZ couplings and $z=M_{Z}^{2} / s ; \sigma_{V V}, \sigma_{V A}$ and $\sigma_{A A}$ are

$$
\begin{gathered}
\sigma_{V V}=e_{e}^{2} e_{F}^{2}+\frac{2 e_{e} e_{F} v_{e} v_{F}}{1-z}+\frac{\left(a_{e}^{2}+v_{e}^{2}\right) v_{F}^{2}}{(1-z)^{2}}, \sigma_{A A}=\frac{\left(a_{e}^{2}+v_{e}^{2}\right) a_{F}^{2}}{(1-z)^{2}} \\
\sigma_{V A}=\frac{2 e_{e} e_{F} a_{e} a_{F}}{1-z}+\frac{4 v_{e} a_{e} v_{F} a_{F}}{(1-z)^{2}}
\end{gathered}
$$

The total production cross sections and forward-backward asymmetries are then

$$
\sigma_{F}=\sigma_{0} N_{c}\left[\frac{1}{2} \beta_{F}\left(3-\beta_{F}^{2}\right) \sigma_{V V}+\beta_{F}^{3} \sigma_{A A}\right] \quad, \quad A_{F}=\frac{3}{4} \beta_{F} \frac{\sigma_{V A}}{\frac{1}{2}\left(3-\beta_{F}^{2}\right) \sigma_{V V}+\beta_{F}^{2} \sigma_{A A}}
$$

The cross sections for mirror (which are the same for sequential) and vector isodoublet heavy leptons are displayed in Fig. 1a for a c.m. energy of $1 \mathrm{TeV}$; the cross sections for heavy quarks are of the same order of magnitude as the one for $E$. As one can see, they are rather large: with $\int \mathcal{L}=100 \mathrm{fb}^{-1}$ one can expect $\sim 10^{3}-10^{4}$ events. The backgrounds (mainly from three vector boson production) have been discussed in [12], and are small compared to the signal. It is therefore clear that the detection of pair-produced heavy leptons with masses close to the kinematical limit should be straightforward at $e^{+} e^{-}$colliders. The angular distributions are shown in Fig. 1b, and one notes that they are symmetric for vector fermions leading to $A_{F B}=0$; for mirror fermions, $A_{F B}$ is sizeable and has opposite sign compared to sequential fermions. The polarization 4 -vectors of the heavy fermions can be measured and would also allow to discriminate between mirror, vector and sequential fermions [12].

Charged fermions can also be pair-produced at $\gamma \gamma$ colliders, the total cross section for unpolarized photon beams is given by

$$
\sigma=3 N_{c}^{f} e_{F}^{4} \sigma_{0} \beta_{F}\left[1+\beta_{F}^{2}+\frac{1-\beta^{4}}{2 \beta} \log \frac{1+\beta}{1-\beta}\right]
$$

It is shown in Fig. 1c for the charged lepton, assuming a fixed c.m energy of 0.8 $\mathrm{TeV}$. Although smaller lepton masses can be probed because of the loss in energy, the $\gamma \gamma$ mode is interesting since the rates can be much larger than in $e^{+} e^{-}$for low $m_{F}$. For quarks, the charge is penalizing and the rates are $N_{c}^{f} e_{Q}^{4}$ times smaller. 
Figure 1: Total production cross sections (a) and angular distributions (b) for the pair production of vector and mirror leptons in $e^{+} e^{-}$collisions at $\sqrt{s}=1 \mathrm{TeV}$, and cross

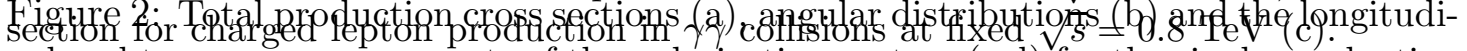
nal and transverse components of the polarization vectors $(c, d)$ for the single production of heavy leptons with $\mathrm{LH}$ and RH mixings in $e^{+} e^{-}$collisions at $\sqrt{s}=1 \mathrm{TeV}$.

In $e^{+} e^{-}$collisions one can also have access to the new fermions via single production in association with their light partners if the mixing is not too small [12]. The process proceeds only via $s$-channel $Z$ exchange in the case of quarks and second/third generation leptons (if inter-generational mixing is neglected); because $\zeta_{L, R}<0.1$, the cross sections are very small and make these states unlikely to be found this way. But for the first generation heavy leptons, one has additional $t$-channel exchanges: $W$ exchange for $N$ and $Z$ exchange for $E$, which increase the cross sections by several orders of magnitude. The analytical expressions are quite involved [12], however, at very high energies, $w=M_{W}^{2} / s$ and $z=M_{Z}^{2} / s$ can be neglected and if one uses $s_{W}^{2} \simeq 1 / 4$ and assumes $\zeta_{L, R}^{\nu N}=\zeta_{L, R}^{e N}=\zeta_{L, R}$, one obtains simple formulae which are good approximations. Using the scaled mass $a=m_{L}^{2} / \mathrm{s}$, one has for $\sigma(L) \equiv \sigma\left(e^{+} e^{-} \rightarrow L \bar{l}\right)$

$$
\begin{gathered}
\sigma\left(L_{L, R}\right)=3 \sigma_{0}\left(\zeta_{L, R}\right)^{2}(1-a) \kappa\left(L_{L, R}\right) \\
\kappa\left(N_{L}\right)=\frac{1}{w}+\frac{1}{3} \frac{a-4}{1-a} \log \frac{1-a+w}{w}, \kappa\left(N_{R}\right)=\frac{-1}{1+w-a}+\frac{a}{1-a} \log \frac{1-a+w}{w} \\
\kappa\left(E_{L}\right)=\kappa\left(E_{R}\right)=\frac{1}{9}\left[\frac{1-a}{z(1-a+z)}-4 \log \frac{1-a+z}{z}\right]
\end{gathered}
$$

The total cross sections are the same for the charge conjugate states, and for Majorana neutrinos it is $\sigma=\sigma(L)+\sigma(\bar{L})[7]$. They are shown in Fig. 2a for all mixing angles taken to be $\zeta_{L, R}=0.1$. The cross sections are very large, especially for $N_{L}$ where they reach the picobarn level. For $E_{L, R}$ they are one order of magnitude smaller, a consequence of the smaller NC couplings compared to CC couplings. For $N_{R}$, the cross section is approximately $10 \mathrm{fb}$ across the entire mass range. For smaller mixing angles the rates have to be scaled correspondingly; even for $E$ and $N_{R}$, requiring 10 events with $\int \mathcal{L}=100 \mathrm{fb}^{-1}$ for $m_{L}=800 \mathrm{GeV}$, one can probe $\zeta$ values one order of magnitude smaller.

The angular distributions are shown in Fig. 2b, and one clearly sees that one can distinguish between neutrinos with LH and RH mixing, and of Dirac or Majorana type. A further distinction can be made by measuring the final polarization; for instance the longitudinal and transverse components of the polarization vectors of $E_{L}$ and $E_{R}$ (which cannot be discriminated by the angular distributions) are practically equal in magnitude but opposite in sign as shown in Fig. 2c/2d.

To fully reconstruct the heavy lepton masses, the best signals consist of an $e^{+} e^{-}$pair and two jets for the charged lepton and an $e^{ \pm}$, a pair of jets and missing momentum for the neutral lepton; the branching ratios are $23 \%$ and $43 \%$ respectively. In the case of $E$, the main backgrounds are: $e^{+} e^{-} \rightarrow e^{+} e^{-} Z \rightarrow e^{+} e^{-} j j$, $e^{+} e^{-} \rightarrow Z Z, e^{+} e^{-} \rightarrow t \bar{t} \rightarrow W^{+} W^{-} j j$ and $\gamma \gamma \rightarrow e^{+} e^{-} q \bar{q}$. In the case of $N$, the 
backgrounds are: $e^{+} e^{-} \rightarrow e \nu W, e^{+} e^{-} \rightarrow W W \rightarrow e^{ \pm} \nu_{j} j$ and $\gamma \gamma \rightarrow e(e) q \bar{q}$. These backgrounds can be eliminated or reduced by applying the following cuts for $N(E)$ production: 1) require one and only one $e^{ \pm}\left(e^{+} e^{-}\right.$pair $\left.), 2\right)$ the invariant mass of the two jets should reconstruct to $\left.M_{W}\left(M_{Z}\right), 3\right)$ the invariant mass of the $e^{+} e^{-}$pair should be large (different from $M_{Z}$ ), 4) the momentum of the neutrino and charged leptons (two charged leptons) should be large, 5) cuts on the angle between the initial electron and the $W(Z)$ boson, and 6) a cut $\cos \theta_{l \nu}\left(\cos \theta_{l l}\right)<0.5$. Optimizing these cuts, no events from heavy flavor production or from the $\gamma \gamma$ backgrounds would survive; the backgrounds from vector boson production can be suppressed to a very low level, while those from single $W / Z$ production can be a bit higher.

A full simulation [12, 30] of the signal and backgrounds has been performed using PYTHIA, for a model detector (an upgraded LEP detector) to quantify the discovery limits that can be obtained. This simulation was done assuming a c.m. energy of $500 \mathrm{GeV}$ and an integrated luminosity of $50 \mathrm{fb}^{-1}$. The signal and background cross sections after applying cuts are shown in Table 2 (note that at $\sqrt{s}=500 \mathrm{GeV}$, the cross sections are practically the same at $1 \mathrm{TeV}$, because the dominant contribution comes from the $t$-channel exchange). The output for heavy leptons with masses of 250,350 and $450 \mathrm{GeV}$ and with $\zeta=0.05$ for $E$ and $\zeta_{L}=0.025$ for $N$ is shown in Fig. 3. For these $\zeta$ values, one can see that the signal peaks stand out clearly from the background events, especially for $m_{L}$ not too close to $\sqrt{s}$. For $m_{E} \sim 450 \mathrm{GeV}$, only slightly smaller $\zeta$ values can be probed, while for $m_{E} \sim 350 \mathrm{GeV}$ one can go down by at least a factor of two. The situation is much more favorable for $N_{L}$, the cross section being one order of magnitude larger. For $m_{L}=350 \mathrm{GeV}$ and requiring that the signal over the square-root of the background is larger than unity, one can probe mixing angles down to $\zeta \sim 0.005$ for neutral leptons and $\zeta \sim 0.03$ for charged leptons. At $\sqrt{s}=1$ $\mathrm{TeV}$, these numbers for $m_{L}$ and $\zeta^{2}$ values can be improved by a factor of two.

Finally, we note that heavy fermions cannot be produced singly at $\gamma \gamma$ colliders (at least in a $2 \rightarrow 2$ process); heavy neutral and charged leptons can be produced in $e \gamma$ collisions in association with massive gauge bosons [31], however only smaller masses can be probed and the rates are not much larger than in $e^{+} e^{-}$collisions.

Table 2: Cross sections for heavy lepton single production and for the main backgrounds at $\sqrt{s}=0.5 \mathrm{TeV}$ after successive applications of cuts; $m_{L}=350 \mathrm{GeV}$ and $\zeta=0.025(0.05)$ are chosen for $N(E)$ and the masses are in $\mathrm{GeV}$.

Figure 3: Reconstructed masses for the singly produced heavy leptons in $e^{+} e^{-} \rightarrow e^{+} e^{-} j j$ (a) and in $e^{+} e^{-} \rightarrow \nu_{e} e j j$ (b) at $\sqrt{s}=0.5 \mathrm{TeV}$, and for the main backgrounds after the application of the cuts displayed in Tab. 2 . 


\subsection{New particles in Left-Right and Extended Models}

In this subsection, we will discuss separately the case of the new particles predicted by Left-Right Models (LRM) based on the symmetry $\mathrm{SU}(2)_{L} \times \mathrm{SU}(2)_{R} \times \mathrm{U}(1)_{B-L}$ 32. In these models, the $\mathrm{LH}$ and $\mathrm{RH}$ fermions transform as doublets under $\mathrm{SU}(2)_{L}$ and $\mathrm{SU}(2)_{R}$ respectively, and therefore each generation contains a $\mathrm{RH}$ neutrino. The extended symmetry leads also to new neutral and charged interactions mediated by heavy $Z^{\prime}$ and $W_{R}$ gauge bosons. These new gauge bosons will mix with the SM $Z$ and $W$ bosons, but the mixing is rather small and can be neglected here. The masses of the new gauge bosons are constrained to be larger than $\sim 0.5$ $\mathrm{TeV}$; however, in models with arbitrary Yukawa couplings $M_{W_{R}}$ can be as low as $300 \mathrm{GeV}$ [23]. The minimal Higgs sector contains doublet and triplet scalar fields which leads to the existence of neutral, charged and also doubly charged $\left(\Delta^{++}\right)$ Higgs bosons. Supersymmetric versions of the LRM have also been considered, and their particle content is much richer than that of the Minimal Supersymmetric SM. In particular, there is a doubly charged fermion, the Higgsino $\tilde{\Delta}^{++}$. We will discuss the manifestation of some of these new particles at $e^{+} e^{-}$colliders.

The heavy RH neutrinos can be produced in pairs at $e^{+} e^{-}$colliders, $e^{+} e^{-} \rightarrow$ $N N$, through the $s$-channel exchange of a heavy $Z^{\prime}$ and the $t$-channel exchange of the RH boson $W_{R}$; for Majorana neutrinos, one has also a $u$-channel $W_{R}$ exchange. This process has been discussed in [8] where the expressions for the cross sections can be found. At a $500 \mathrm{GeV} e^{+} e^{-}$collider, they are shown in Fig. 4 for $g_{L}=g_{R}$ and $M_{Z^{\prime}}=M_{W_{R}}=1$ and $1.5 \mathrm{TeV}$ and for Dirac and Majorana neutrinos. The cross section for Dirac is slightly larger than for Majorana neutrinos, especially close to the kinematical limit due the well-known $\beta^{3}$ suppression factor for the latter.

Figure 4: Total cross sections for $e^{+} e^{-} \rightarrow N N$ production at a $500 \mathrm{GeV} e^{+} e^{-}$collider.

To probe the Majorana or Dirac nature of the heavy neutrino, one can use the angular distributions, which are different. $N$ will decay through $\mathrm{RH}$ charged currents into a charged lepton and two-jets. Requiring 20 events with $\mathcal{L}=20 \mathrm{fb}^{-1}$, one can probe masses of the order of 230 (200) for Dirac (Majorana) neutrinos for $M_{W_{R}}=1.5 \mathrm{TeV}$. Note that heavy neutrinos can be also singly produced in $e P$ collisions [8] through $W_{R}$ exchange, as will be discussed later.

One can look at the situation the other way around, and try to produce $W$ bosons through the exchange of the heavy neutrinos. An interesting possibility is the production of like sign $W$ pairs in $e^{-} e^{-}$collisions 33]. This process, if it exists would signal the existence of new $|\Delta L|=2$ interactions which may manifest themselves as Majorana masses for neutrinos. However, it is difficult to generate a large cross section for this reaction while simultaneously satisfying the constraint of tree-level unitarity at large values of $s$ and the bounds on the effective neutrino mass arising from the lack of observation of neutrinoless double beta decay. In 
the LRM [32], as a result of the see-saw mechanism used to generate small masses for the ordinary LH neutrinos, these difficulties can be easily circumvented by considering the reaction $e^{-} e^{-} \rightarrow W_{R}^{-} W_{R}^{-}$.

The amplitude for $e^{-} e^{-} \rightarrow W_{R}^{-} W_{R}^{-}$gets both $t$ - and $u$-channel contributions from the exchange of the heavy RH neutrino $N$, as well as an $s$-channel contribution from the exchange of the doubly-charged Higgs boson $\Delta$ with mass $M_{\Delta}$ ( $W-W_{R}$ mixing is neglected here). Since the $e^{-} e^{-} \Delta$ coupling is proportional to $M_{N}$ and the $e^{-} N W_{R}$ coupling is chiral, the total amplitude is proportional to $M_{N}$. Thus, as the Majorana mass of $N$ vanishes so does the amplitude; this is expected since the $|\Delta L|=2$ interaction is generated by the Majorana mass term.

At energies of $\sqrt{s}=0.5-1.5 \mathrm{TeV}$, the cross section for $e^{-} e^{-} \rightarrow W_{R}^{-} W_{R}^{-}$is quite large, of order a few picobarns, is fairly sensitive to the values of $M_{N}$ and $M_{\Delta}$, and has a rather flat angular distribution. The $\Delta$ boson may appear as an $s$-channel resonance depending upon the value of $\sqrt{s}$. Unfortunately, the "reach" is rather limited since we are restricted to $M_{W_{R}}<\sqrt{s} / 2$ values, and since $M_{W_{R}}$ is heavier than $0.5 \mathrm{TeV}$ and $W_{R}$ pair production would be not kinematically accessible at these energies. One has therefore to consider [34] the possibility of single production of $W_{R}$ via the reaction $e^{-} e^{-} \rightarrow W_{R}^{-}\left(W_{R}^{-}\right)^{*} \rightarrow W_{R}^{-} j j$. We limit ourselves to this $j j$ mode to allow for the possibility that $M_{N}>M_{W_{R}}$ in which case the $W_{R}$ can only decay to jets barring the existence of exotic particles.

Allowing one of the $W_{R}$ 's to be off-shell results in a substantial reduction in the cross section from the on-shell case to the level of a few $\mathrm{fb}$, which implies that machine luminosities in the range of $\mathcal{L}=100-200 \mathrm{fb}^{-1}$ are required to make use of this channel. The total event rates for the reaction is shown in Fig. 5, in which we have set $\kappa=g_{R} / g_{L}=1$ and scaled by an integrated luminosity of $100 \mathrm{fb}^{-1}$. Fig. $5 \mathrm{a}$ shows the number of expected $W_{R}+j j$ events, as a function of $M_{W_{R}}$, at a $\sqrt{s}=1$ $\mathrm{TeV} e^{-} e^{-}$collider for different choices of $M_{N}$ and $M_{\Delta}$. The results are seen to be quite sensitive to the values of these masses. In Fig. $5 \mathrm{~b}(\mathrm{c})$, we fix $M_{W_{R}}=700 \mathrm{GeV}$ and plot the event rate as a function of $M_{N}\left(M_{\Delta}\right)$ for various values of $M_{\Delta}\left(M_{N}\right)$. Typically, we see that one can obtain rates of the order of several hundred events a year, except near the $\Delta$ resonance (where the rates are very large) or when $M_{N}$ is small (the cross section vanishes for $M_{N}=0$ ). For most choices of the input masses, one obtains extremely flat angular distributions, except when $N$ is light in

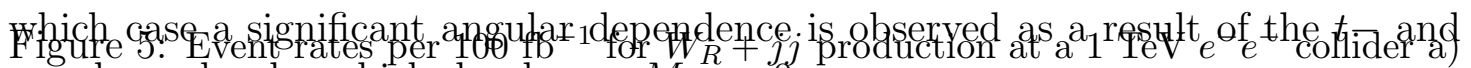

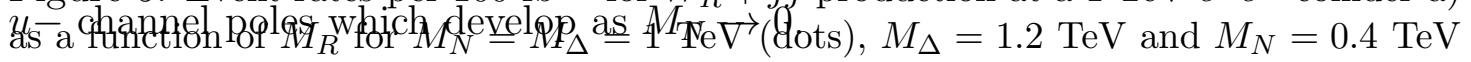
(dashes), $M_{\Delta}=0.3$ and $M_{N}=0.1 \mathrm{TeV}$ (dash-dots), $M_{\Delta}=2, M_{N}=0.6 \mathrm{TeV}$ (solid), or $M_{\Delta}=1.8$ and $M_{N}=0.6 \mathrm{TeV}$ (square dots); (b) with $M_{R}=700 \mathrm{GeV}$ fixed as a function of $M_{N}$ for $M_{\Delta}=0.3(0.6,1.2,1.5,2) \mathrm{TeV}$ corresponding to the dotted(dashed, dashdotted, solid, square-dotted) curve; (c) as a function of $M_{\Delta}$ for $M_{N}=0.2(0.5,0.8,1.2,1.5)$ $\mathrm{TeV}$ corresponding to the dotted(dashed, dash-dotted, solid, square-dotted) curve. 
As mentioned previously, heavy neutrinos may be observed indirectly outside the LRM. One could assume the spontaneous breakdown of e.g. the $E_{6}$ group via the chain $E_{6} \rightarrow S O(10) \rightarrow \ldots$ down to the $\mathrm{SM}$ group, where the $\mathrm{RH} W_{R}$ bosons (and the doubly charged Higgs bosons) are too heavy but the two additional isosinglet neutrinos have masses in the 1-10 TeV range. These Majorana neutrinos will mix with light neutrinos, and could yield the presently observed mass spectrum [36]. It has been shown [36] that this scenario is open to experimental detection through the process $e^{-} e^{-} \rightarrow W^{-} W^{-}$, where at least the lightest of the two heavy neutrinos is exchanged. In contrast to the case where $2 W_{R}$ are produced, this process can be observed even at a $0.5 \mathrm{TeV}$ electron-electron collider.

In the kinematic regime well above the $W$ mass, but below that of the heavy neutrino, where no doubly-charged Higgs contribution is needed for unitarity reasons (letting us avoid the uncertain couplings of an $L=2$ state to a flavorless boson), a characteristic energy dependence $\sim s^{2}$ is shown in Fig. 6. The crosssection band displayed there is bounded by the known limits on heavy-neutrino mixing from rare decay processes and on lepton universality evidence.

Figure 6: Cross-section range for the process $e^{-} e^{-} \rightarrow W^{-} W^{-}$via TeV-mass Majorana neutrino exchange. The curves indicate extreme values of the neutrino mixing parameters allowed by present data.

Note that the spectacular back-to-back $W$ pair decays permit effective background suppression [37, 38], so that even a moderate signal may well lead to a convincing discovery; in particular, a change in incoming electron helicity will eliminate any signal, providing a further test for its legitimacy. This is in contrast to the classic discovery channel for light Majorana neutrinos, neutrinoless double beta decay: here, the heavy masses lead to severe signal suppression, and there is no criterion telling a signal due to light-neutrino exchange from one due to heavy neutrinos 36.

Finally, let us consider the Higgs sector and the supersymmetric version of the LRM [35]. A particularly suitable signal of the model is offered by the triplet higgsino $\tilde{\Delta}^{++}$, a Dirac fermion consisting of the fermion components of the triplet Higgs fields. As being doubly charged it does not mix with other particles, and consequently its mass is given by a single parameter. The decay modes of the $\tilde{\Delta}^{++}$ are very limited, since it carries two units of lepton number and it does not couple to quarks. The nonconservation of the separate lepton numbers $L_{e}, L_{\mu}$, and $L_{\tau}$ of the $\tilde{\Delta}^{++}$couplings is a very special signature which can be studied in slepton pair production where one of the reaction amplitudes includes $\tilde{\Delta}^{--}$exchange.

The allowed decay modes of the triplet higgsino are $\tilde{\Delta}^{++} \rightarrow \Delta^{++} \lambda^{0}, \Delta^{+} \lambda^{+}$, $\tilde{\Delta}^{+} W_{R}^{+}$, and $\tilde{l}^{+} l^{+}$, where $\lambda^{\prime}$ 's represent the fields in the LRM bi-doublet representation. In large regions of the parameter space, the kinematically favoured decay mode is $\tilde{\Delta}^{++} \rightarrow \tilde{l}^{+} l^{+}$(this is of course the case only when $m_{\tilde{l}^{+}}<m_{\tilde{\Delta}^{++}}$, at least 
for some slepton flavour, which we will assume in the following). If the mass of the triplet Higgs $\Delta$ is of the order of the $\mathrm{SU}(2)_{R}$ breaking scale, the first two decay channels are forbidden energetically in the case of relatively light triplet higgsinos. For the same reason the channel $\tilde{\Delta}^{+} W_{R}^{+}$is kinematically disfavoured, since the $W_{R}$ mass is expected to be above $0.5 \mathrm{TeV}$ [23]. The decay channel $\tilde{\Delta}^{+} W^{+}$is supressed by the small $W-W_{R}$ mixing. In the following we will assume that $\tilde{\Delta}^{++}$and its charge conjugate state $\tilde{\Delta}^{--}$decay $100 \%$ of the time into the $\tilde{l} l$ final states. The charged sleptons $\tilde{l}$ can decay either to a charged lepton of the same flavour plus a neutralino, to a neutrino plus a chargino, or to a charged gauge boson plus a sneutrino:

$$
\tilde{l}^{+} \rightarrow l^{+}+\tilde{\chi}_{i}^{0}, \quad \tilde{l}^{+} \rightarrow \nu+\tilde{\chi}_{i}^{+}, \quad \tilde{l}^{+} \rightarrow W^{+}+\tilde{\nu}
$$

Which of the various decay channels is the dominant one depends on the mass of the decaying slepton. The triplet higgsinos can be produced in the next generation linear electron colliders in the $e^{+} e^{-}, e^{-} e^{-}, e^{-} \gamma$ and $\gamma \gamma$ collision modes:

$$
e^{+} e^{-} \rightarrow \tilde{\Delta}^{++} \tilde{\Delta}^{--}, e^{-} e^{-} \rightarrow \tilde{\chi}^{0} \tilde{\Delta}^{--}, \gamma e^{-} \rightarrow \tilde{l}^{+} \tilde{\Delta}^{--}, \gamma \gamma \rightarrow \tilde{\Delta}^{++} \tilde{\Delta}^{--}
$$

All these reactions have a clean experimental signature: a few hard leptons and missing energy, the background from other processes are thus rather small. The largest cross section is obtained for the $\gamma \gamma \rightarrow \tilde{\Delta}^{++} \tilde{\Delta}^{--}$process; the expression is given in eq. (9) and because of the double charge, it is enhanced by a factor 16 compared to the case of a heavy charged lepton for which it is shown in Fig. 1c.

Finally, the enlarged particle content of the Supersymmetric LRM also effects other processes such as the production of slepton pairs. First, the number of neutralinos in the $t$-channel production is larger because of the additional neutral fermions in both the gauge and Higgs sectors. A second difference with the Minimal Supersymmetric SM case is that there is a new $u$-channel diagram due to the doubly charged higgsino discussed above. The combination of these new contributions leads to an increase in the production cross section for slepton pairs in $e^{+} e^{-}$in comparison to the Minimal Supersymmetric SM results 35.

\subsection{Production in $e P$ Collisions}

Heavy leptons of the first generation can be singly produced in $e P$ collisions [39, 40] through $t$-channel exchange of a $W$ boson for the neutral and a $Z$ boson for the charged leptons. One can also have heavy quark production but the backgrounds are stronger and this process will not be considered here. For polarized $e$ beams beams, the differential cross section $\mathrm{d} \sigma / \mathrm{d} x \mathrm{~d} y$ for $e_{L, R} P \rightarrow L X\left(a=m_{L}^{2} / \hat{s}\right)$ is

$$
\begin{gathered}
\frac{d \sigma}{d x d y}=\frac{G_{F}^{2} M_{W}^{4}}{2 \pi} \hat{s} \sum_{j, q=L, R} \zeta_{j}^{2}\left[A_{j q}^{L}\left(Q^{2}\right) H_{j q}(x, y) q\left(x, Q^{2}\right)+\bar{A}_{j q}^{L}\left(Q^{2}\right) \bar{H}_{j q}(x, y) \bar{q}\left(x, Q^{2}\right)\right] \\
H_{L L}(x, y)=\bar{H}_{L R}(x, y)=H_{R R}(x, y)=\bar{H}_{R L}(x, y)=(1-a) \\
H_{L R}(x, y)=\bar{H}_{L L}(x, y)=H_{R L}(x, y)=\bar{H}_{R R}(x, y)=(1-y-a)(1-y)
\end{gathered}
$$


Using $a_{q}=2 I_{q}^{3 L}$ and $v_{q}=\left(2 I_{q}^{3 L}-4 Q_{q} s_{W}^{2}\right)$ the expressions of $A^{L}$ and $\bar{A}^{L}$ are

$A_{j q}^{N}=\bar{A}_{j q}^{N}=\left(Q^{2}+M_{W}^{2}\right)^{-2}, A_{L q}^{E}\left(\bar{A}_{L q}^{E}\right)=\bar{A}_{R q}^{E}\left(A_{R q}^{E}\right)=\left(Q^{2}+M_{Z}^{2}\right)^{-2}\left(v_{q} \pm a_{q}\right)^{2} /\left(16 c_{W}^{4}\right)$

The cross sections are shown in Fig. 7 for $N$ and $E$ production with $\zeta_{L, R}=0.1$, at a c.m energy $\sqrt{s}=1.2 \mathrm{TeV}$. The largest cross section is obtained in the case of $N_{L}$, closely followed by the one for $N_{R}$ production; the cross sections for $E_{L, R}$ are a bit less than one order of magnitude smaller. The best signature is $e^{-}$-jet-jet (see the branching ratios above); for Majorana neutrinos, also $e^{+} W^{-}$final states would be observed [7]. Requiring 20 events and assuming a luminosity of $2 \mathrm{fb}^{-1}$, one can probe masses up to $700 \mathrm{GeV}$ for $N$ and $550 \mathrm{GeV}$ for $E$, assuming $\zeta=0.1$. Note that, together with the $y$ distributions, the difference between the longitudinal and transverse components of the polarizations vectors of the final leptons can be used to discriminate between particles with LH or RH mixing [39].

For the neutral leptons, the main backgrounds are $W / Z$ production, heavy flavor production and higher order QCD radiation; beam polarization cannot be used to reduce them. A detailed analysis 40 has been performed to obtain allowed regions in the $m_{N}-\zeta$ plane. For $\zeta=0.1$ it has been found that the search reaches $\sim 800 \mathrm{GeV}$ at LEP $\times$ LHC (with $\sqrt{s}=1.3 \mathrm{TeV}$ and $\mathcal{L}=2 \mathrm{fb}^{-1}$ ). For HERA $\left(\sqrt{s}=314 \mathrm{GeV}\right.$ and $\left.\mathcal{L}=200 \mathrm{pb}^{-1}\right)$ and a HERA upgrade $(\sqrt{s}=450 \mathrm{GeV}$ and $\left.\mathcal{L}=4 \mathrm{fb}^{-1}\right)$, one reaches the limits 160 and $320 \mathrm{GeV}$ respectively.

Figure 7: Total production cross sections for the single production of heavy leptons in $e P$ collisions at $\sqrt{s}=1.2 \mathrm{TeV}$.

In the Left-Right symmetric models discussed previously, heavy neutrinos of the first generation can be produced in $e P$ collisions via $t$-channel $W_{R}$ exchange (this process is somewhat complementary to searches for $W_{R}$ at the Tevatron in which $W_{R} \rightarrow N e$ and the $N$ decays within the detector). The reach for this process was explored in a detailed Monte Carlo study [40] under the assumptions that $g_{R}=g_{L}$ and that the CKM matrices in the LH and RH sectors are the same. RH beam polarization can be used to reduce backgrounds, and Majorana decay signatures for $N$ are required as tags. For HERA, a HERA upgrade and $\mathrm{LEP} \times \mathrm{LHC}$, the discovery reach that has been found approximately corresponds to the following regions in the $m_{N}-M_{W_{R}}$ plane: $m_{N}+0.34 M_{W_{R}}<276 \mathrm{GeV}$, $m_{N}+0.20 M_{W_{R}}<394 \mathrm{GeV}$, and $m_{N}+0.38 M_{W_{R}}<1090 \mathrm{GeV}$.

\subsection{Production at $p p$ Colliders}

Proton colliders are ideal machines to search for heavy quarks [11]. The pair production subprocesses are gluon-gluon fusion, $g g \rightarrow Q \bar{Q}$ and quark-antiquark annihilation, $q \bar{q} \rightarrow Q \bar{Q}$, with the gluon fusion subprocess being by far dominant. 
The tree-level partonic cross sections are well-known 42

$$
\begin{aligned}
\sigma_{g g}(\hat{s}) & =\frac{\pi \alpha_{S}^{2}}{3 \hat{s}}\left[\left(1+a+\frac{a^{2}}{16}\right) \log \frac{1+\beta}{1-\beta}-\beta\left(\frac{7}{4}+\frac{31}{16} a\right)\right] \\
\sigma_{q q}(\hat{s}) & =\frac{8 \pi \alpha_{S}^{2}}{27 \hat{s}} \beta\left(1+\frac{1}{2} a\right)
\end{aligned}
$$

with $\beta$ the velocity of the quark, $\beta^{2}=1-a=1-4 m_{Q}^{2} / \hat{s}$. The total cross sections are obtained by integrating over the gluon and quark luminosities. One can use the previous tree level formulae in a way so as to reproduce the full one-loop corrected $Q \bar{Q}$ cross-section [43] in the limit where the $P_{T}^{j}$ of the additional jet tends to zero. The shower approximation can be used for this purpose [44] and the tree-level $Q \bar{Q}+1$ jet differential cross-section can be replaced by

$$
d \sigma(Q \bar{Q} j) \longrightarrow d \sigma(Q \bar{Q} j) \times\left[1-\exp \left(-C P_{T}^{j 2}\right)\right]
$$

with the constant $C$ properly chosen to reproduce the cross section at $\mathcal{O}\left(\alpha_{\int}^{\ni}\right)$. The obtained result is shown in Fig. 8 at the LHC for two c.m. energies: $\sqrt{s}=10$ and $14 \mathrm{TeV}$. The HMRS(B) structure functions 445] are used and the scale $Q^{2}$ in the evaluation of the structure functions and $\alpha_{S}$ is chosen to be equal to $Q^{2}=E_{T}^{2}=$ $m_{Q}^{2}+P_{T}^{2}(Q)$.

The cross section is very large, and one sees that at $\sqrt{s}=14 \mathrm{TeV}$, even for $m_{Q} \sim 1 \mathrm{TeV}$, it is at the level of $0.1 \mathrm{pb}$ which leads to 1000 events even at a moderate luminosity of $10 \mathrm{fb}^{-1}$. The best signature makes use of the "goldplatted" decay mode where the heavy quark decays into its light partner and a $Z$ boson, with the latter subsequently decaying into two charged leptons $l=e, \mu$. This leads to the spectacular final state

$$
p p \rightarrow Q \bar{Q} \rightarrow(q Z)(\bar{q} Z) \rightarrow\left(j l^{+} l^{-}\right)\left(j l^{+} l^{-}\right)
$$

which has a somewhat small branching ratio: $\sim 5.10^{-4}$ for large quark masses. Allowing one of the $Z$ bosons to decay into jets or neutrinos, or one of the $Q$ 's to decay into a quark and a $\mathrm{W}$ boson (which then decays into jets or leptons) will drastically enhance the cross section times branching ratio rate. The obtained signals involve at least two leptons and are still very interesting. It is therefore very likely that heavy quarks, with masses up to $\sim 1 \mathrm{TeV}$ can be found at LHC.

Heavy leptons can also be produced in $p p$ collisions. The processes are: the Drell-Yan mechanism $q \bar{q} \rightarrow L \bar{L}$ with $\gamma / Z$ exchange for $E$ and only $Z$ exchange for $N$, the gluon-fusion mechanism $g g \rightarrow L \bar{L}$ which proceeds through quark loops and $Z+$ Higgs boson exchange, and for the charged lepton the $\gamma \gamma$ fusion process $\gamma \gamma \rightarrow L^{+} L^{-} X$. In addition, one has associated production of $N$ and $E$ in the Drell-Yan process $q q \rightarrow W^{*} \rightarrow N E$. For singlet neutrinos, since they have no weak charges, none of these processes is at work; one has therefore to produce 
them through mixing and since the angles are small, it is rather difficult to find these states at $p p$ colliders. The total cross sections for the charged leptons 411 are shown in Fig. 9 at LHC with $\sqrt{s}=14 \mathrm{TeV}$ as a function of $m_{L}$; the ones for neutral leptons (excluding the $\gamma \gamma$ process) which fully couple to the $Z$ should be of the same order of magnitude. They have been obtained using the $\operatorname{HMRS}(\mathrm{B})$ structure functions at the scale $\mu^{2}=\hat{s} / 4 ; \alpha_{S}$ is evaluated at the two loop level in the $\overline{M S}$ scheme with $\Lambda \frac{4}{M S}=0.19 \mathrm{GeV}$. For the $g g$ fusion, we assumed only three generations of quarks, and set $m_{t}=175$ and $M_{H}=300 \mathrm{GeV}$. Fig. 9 shows that the Drell-Yan process has the largest cross section for small $m_{L}, \sim 1 \mathrm{pb}$ for $m_{L}=100 \mathrm{GeV}$, but it falls to $\sim 0.1 \mathrm{pb}$ for $m_{L}=700 \mathrm{GeV}$. The $g g$ fusion cross section falls less rapidly and dominates for $m_{L}>500 \mathrm{GeV}$. Requiring 100 events for $\int \mathcal{L}=10(100)$ $\mathrm{fb}^{-1}$, one can reach lepton masses of the order of 400 (700) GeV. The lower curves represent the inelastic (solid), elastic (dashed) and semi-elastic (dot-dashed) $\gamma \gamma$ cross sections; they are three orders of magnitude smaller. However, these processes might prove useful in confirming the signal.

Figure 8: Total production cross sections (in nb) for heavy quarks at LHC for two c.m. energy values: $\sqrt{s}=10$ and $14 \mathrm{TeV}$.

Figure 9: Total production cross sections for heavy charged leptons at LHC with $\sqrt{s}=$ $14 \mathrm{TeV}$, in the Drell-Yan, the gluon-fusion and the $\gamma \gamma$ fusion mechanisms.

\section{$3 \quad$ Excited Fermions}

\subsection{Introduction, Lagrangians and Decay Modes}

In this study, we will assume that the excited fermions [14, 15 have spin and isospin $1 / 2$ to limit the number of parameters (higher spin and isospin have been discussed in 46]). Furthermore, to accommodate the fact that the excited states are much heavier than the ordinary fermions we will assume that they get their masses prior to $\mathrm{SU}(2)_{L} \times \mathrm{U}(1)_{Y}$ breaking and hence, their couplings to the gauge fields are vector-like. Therefore, denoting the excited fermion doublet by $F^{\star}=F_{L}^{\star}+F_{R}^{\star}$, the $F^{*} F^{*}$-gauge boson interaction Lagrangian is

$$
\mathcal{L}_{f^{\star} f^{\star}}=\overline{F^{\star}} \gamma^{\mu}\left[g(\vec{\tau} / 2) \vec{W}_{\mu}+g^{\prime}(Y / 2) B_{\mu}+g_{S}(\vec{\lambda} / 2) \vec{G}_{\mu}\right] F^{\star}
$$

where $\vec{\tau}$ are the Pauli matrices, $Y$ the weak hypercharge $(-1$ for leptons and $1 / 3$ for quarks) and $g, g^{\prime}$ the usual weak couplings constants $g=e / \sin \theta_{W}$ and $g^{\prime}=$ $e / \cos \theta_{W} ; \vec{\lambda}$ are the Gell-Mann matrices and $g_{S}$ the strong coupling constant. Note that form factors and contact interactions may be present, they will be discussed in the context of hadron colliders where they play an important role. 
The Lagrangian describing the transition between excited fermions and ordinary fermions should respect a chiral symmetry in order to protect the light leptons from radiatively acquiring a large anomalous magnetic moment [47]. This means that only the right-handed part of the excited fermions takes part in the generalized magnetic de-excitation and we have [14, 15]:

$$
\mathcal{L}_{f f^{\star}}=(1 / 2 \Lambda) \overrightarrow{F^{\star}} \sigma^{\mu \nu}\left[g f(\vec{\tau} / 2) \vec{W}_{\mu \nu}+g^{\prime} f^{\prime}(Y / 2) B_{\mu \nu}+g_{S} f_{S}(\vec{\lambda} / 2) \overrightarrow{G_{\mu \nu}}\right] f_{L}+h . c
$$

$\Lambda$ is the scale of substructure which we will take of the order of $1 \mathrm{TeV}$, while the $f^{\prime} \mathrm{s}$ are weight factors associated with the three gauge groups; the tensors $V_{\mu \nu}$ are the fully gauge-invariant field tensors. We will set $f=f^{\prime}=f_{S}$ : this not only reduces the number of parameters so that a more predictive analysis can be conducted, but also is more natural since for $f=f^{\prime}$ the excited neutrino has no tree-level electromagnetic couplings [14, 15]. Therefore, apart from the masses of the excited fermions, the only other parameter is the strength of the de-excitation $f / \Lambda$.

We will only consider masses for the excited fermions above $M_{Z}$ since smaller masses will be probed at LEP2. In this case the two body decays into $W / Z$ bosons and light fermions are kinematically allowed. The decay widths for $f^{*} \rightarrow V f$ where $V=\gamma, Z, W$ are given by

$$
\Gamma\left(f^{\star} \rightarrow f^{\left({ }^{\prime}\right)} V\right)=\frac{\alpha}{4} \frac{m_{f^{*}}^{3}}{\Lambda^{2}} f_{V}^{2}\left(1-\frac{M_{V}^{2}}{m_{f^{*}}^{2}}\right)^{2}\left(1+\frac{M_{V}^{2}}{2 m_{f^{*}}^{2}}\right)
$$

with $f_{\gamma}=e_{f} f, f_{W}=f /\left(\sqrt{2} s_{W}\right)$ and $f_{Z}=\left(4 I_{f}^{3}-4 e_{f} s_{W}^{2}\right) f /\left(4 s_{W} c_{W}\right)$. For excited quarks, there is also the very important decay $q^{\star} \rightarrow q g$; the width is given by the previous equation with $\alpha \rightarrow 4 \alpha_{S} / 3$ and $f_{V}=f$. The $f^{\star}$ have very narrow widths: for $m_{f^{\star}}=500 \mathrm{GeV}$ and $\Lambda / f=1 \mathrm{TeV}$ the total width of the $e^{*}$ is less than $1 \mathrm{GeV}$. For masses much larger than $M_{Z}$, the branching ratios are unambiguously predicted since they do not depend on $m_{f^{\star}}$ and $\Lambda$; they are given in Table 3.

Table 3: Branching ratios for the decays of excited fermions (in \%) for large masses.

\begin{tabular}{||c||c|c|c|c||}
\hline$f^{*}$ & $f^{*} \rightarrow f \gamma$ & $f^{*} \rightarrow f Z$ & $f^{*} \rightarrow f W$ & $f^{*} \rightarrow f g$ \\
\hline \hline$\nu^{\star}$ & 0 & 39. & 61. & 0. \\
\hline$e^{\star}$ & 29. & 11. & 60. & 0. \\
\hline$u^{\star}$ & 2. & 3. & 10. & 85. \\
\hline$d^{\star}$ & 0.5 & 5. & 10. & 84.5 \\
\hline
\end{tabular}

As one can see, the electromagnetic decay of charged excited fermions is not the dominant one. For $e^{*}$, it is just about $30 \%$ compared to almost $100 \%$ for masses smaller than $M_{W}$. For quarks, this electromagnetic decay, which would constitute the cleanest way for "tracking" these particles, is a very small fraction of all decays; therefore, relying on this mode leads to a considerable loss of events. Nevertheless, it constitutes a very characteristic signature of excited fermions and could help to disentangle them from the exotic fermions discussed previously. 


\subsection{Production at $e^{+} e^{-}$Colliders}

Pair Production. If kinematically allowed, excited fermions can be pair-produced without any suppression due to the factor $f / \Lambda$ (form factors might be present, though). In $e^{+} e^{-}$collisions the reaction proceeds through $\gamma$ and $Z s$-channel exchange for charged fermions, whereas for excited neutrinos there is only a $Z$ exchange for $f=f^{\prime}$; the charged excited fermions can also be pair produced in the $\gamma \gamma$ mode of the collider. The differential and total cross sections are the same as for the vector-like exotic fermions discussed previously. The only difference will be in the decay modes: while exotic fermions will decay only to $W / Z$ and light fermions, excited charged fermions have the electromagnetic decay and excited quarks will dominantly decay into quarks plus gluons; see Tab. 3. Since the production rates are rather large (see the previous discussion on exotic fermions, in particular Figs. 1), all these final states can be easily searched for in the clean environment of $e^{+} e^{-}$colliders, and the discovery limits that can be reached will be nearly the kinematical limit of $m_{f^{*}} \sim \sqrt{s} / 2$ [15].

Single Production. Owing to the special coupling of the excited fermions to their light partners, one can also have single production of the excited particles. Hence, in principle, $f^{*}$ masses up to the total c.m. energy of the collider can be probed. However, the rates depend on the parameter $f / \Lambda$ which measures the strength of the transition. We will consider single production at $e^{+} e^{-}$colliders in the three modes: $e^{+} e^{-}, e^{-} \gamma, \gamma \gamma$. We will take $\Lambda / f=1 \mathrm{TeV}$ in our numerical analysis.

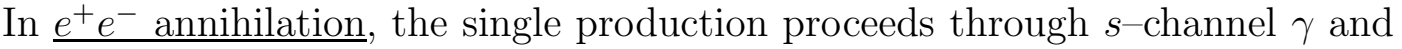
$Z$ exchange for all excited fermions. For the first generation of excited leptons, one has substantial contributions due to additional $t$-channel diagrams: $W$ exchange in the case of the $\nu^{*} ; Z$ and the important $\gamma$ exchange in the case of the $e^{*}$. These processes should be compared to the single production of exotic heavy fermions; however, in the latter case, there is no photon exchange and the couplings are not of the magnetic type. The total section for the single production of $\nu_{e}^{*}$, which is the same for $\overline{\nu_{e}} \nu^{\star}$ is 15

$$
\begin{aligned}
\sigma & =\sigma_{0} \frac{3 s}{4 \Lambda^{2}} \beta\left\{\left(1-\frac{2}{3} \beta\right) \beta A_{\nu}+\frac{f_{W}^{2}}{2 s_{W}^{2}}\left[(2 w+\beta) \log \left(1+\frac{\beta}{w}\right)-2 \beta\right]\right. \\
& \left.+\frac{2 f_{W}}{\sqrt{2} s_{W}}\left(e_{e} f_{\gamma}+\frac{a_{e}+v_{e}}{1-z} f_{Z}\right)\left[w\left(1+\frac{w}{\beta}\right) \log \left(1+\frac{\beta}{w}\right)-\frac{1}{2}(\beta+2 w)\right]\right\}
\end{aligned}
$$

where $\beta=\left(1-m_{\nu^{*}}^{2} / s\right), w=M_{W}^{2} / s, z=M_{Z}^{2} / s$ and $A_{f}$ (with $f=\nu$ ) is given by

$$
A_{f}=e_{e}^{2} e_{f}^{2}+\frac{2 e_{e} v_{e} f_{\gamma} f_{Z}}{1-z}+\frac{\left(a_{e}^{2}+v_{e}^{2}\right) f_{Z}^{2}}{(1-z)^{2}}
$$

These formulae may be used for all other flavours (except for the $e^{\star}$ ) by setting $f_{W}=0$ and by including the colour factor for quarks. For the $e^{\star}$, the expression 
of the total cross section is quite involved and can be found in [15]; a very good approximation is to consider only the $s$ and $t$ channel photon exchange, where the much simpler expression is given by [15

$$
\sigma=\sigma_{0} \frac{3 s}{4 \Lambda^{2}} f_{\gamma}^{2} \beta\left[\left(1-\frac{2}{3} \beta\right) \beta-\beta-3+\frac{1+\beta^{2}}{\beta} \log \left(\frac{s}{m_{e}^{2}} \frac{\beta^{2}}{(1-\beta)^{2}}\right)\right]
$$

In Figs. 10a/b we show the total cross sections at a c.m. energy of $1 \mathrm{TeV}$. The largest production rate occurs for the $e^{\star}$ due to the $t$-channel photon exchange: compared to the other charged leptons this has a two-order of magnitude enhancement. The same is true for the $\nu_{e}^{\star}$ as compared to the other excited neutrinos as a result of the $W$ exchange. Charged excited fermions should be looked for by exploiting their electromagnetic decays; requiring a cut on the transverse momentum of the photon to be larger than $\sim 20 \mathrm{GeV}$ together with a rapidity cut of $\sim 2$ should be sufficient to suppress potential backgrounds from radiative QED processes. For the $e^{*}$, slightly more severe cuts should be applied to further reduce the Bhabha background; one can also use the $e^{+} e^{-} j j$ final states, similarly to what has been discussed in the case of heavy charged leptons. For excited neutrinos, the situation is also similar to that of exotic neutrinos (although the distributions are different) and one has to look for $e \nu j j$ events. A detailed analysis of the background has not been performed here; requiring 20 events to establish a signal for $\int \mathcal{L}=100 \mathrm{fb}^{-1}$,

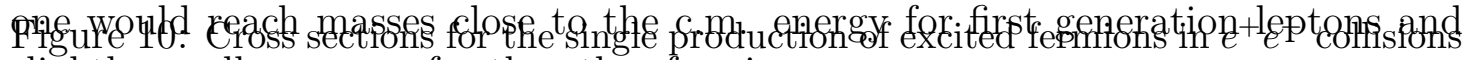

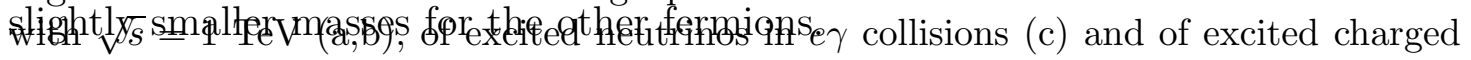
fermions in $\gamma \gamma$ collisions (d). 
In $e \gamma$ collisions, excited leptons of the first generation can be also singly produced. In fact, a motivation for running in this mode would be the production of $e^{\star}$ as a resonance, thus (if this particle exists) turning the machine into an $e^{\star}$ factory. The cross-section after integrating over the Breit-Wigner resonance is 48

$$
\sigma\left(\gamma e \rightarrow e^{\star}\right)=\frac{8 \pi^{2}}{m_{e^{\star}}^{2}} \frac{\Gamma\left(e^{\star} \rightarrow e \gamma\right)}{m_{e^{\star}}}
$$

The best channel to detect this particle is the electromagnetic decay, although there is a potential large background from Compton scattering. However, the bulk of these background events is along the beam direction. Moreover, the produced electron from Compton scattering flies opposite to the initial electron from the beam whereas the electron from the signal has a spherical distribution. Requiring an observation of 20 events over the background $e \gamma \rightarrow e \gamma$, one can reach a limit on the scale $\Lambda$ of about $200 \mathrm{TeV}$, provided that the mass is below the kinematical limit which is approximately $900 \mathrm{GeV}$ for a $1 \mathrm{TeV} e^{+} e^{-}$collider.

In the $e \gamma$ mode, one can also search for the $\nu_{e}^{\star}$ which can be produced in association with a $W, e^{-} \gamma \rightarrow \nu_{e} W^{-}$. Therefore one can in principle reach masses of the order of $800 \mathrm{GeV}$. Fig. 10c shows the total cross section for various values of $m_{e^{*}}$ (which can be exchanged in the $s$-channel) and even for infinite $m_{e^{*}}$, where it is the smallest, the cross section is larger than in $e^{+} e^{-}$collisions. For the signature, one can use the decay into $e^{-} W^{+}$and by letting both the primary $W^{-}$and the decay $W^{+}$go into jets which seems to be background-free. The bounds on $\Lambda$ which can be reached when requiring 20 events, are $\mathcal{O}(100 \mathrm{TeV})$ for $m_{\nu_{e}^{*}}$ smaller than $\sim 800 \mathrm{GeV}$, assuming the same luminosity as in the $e^{+} e^{-}$mode.

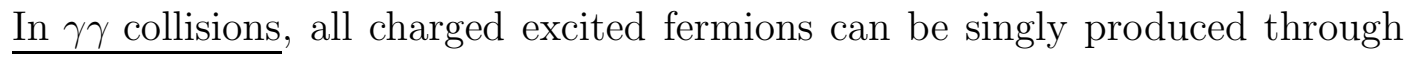
two $t$-channel exchanges: one involving $f^{*}$ and the other $f$. The latter gives a very large contribution, similarly to the $t$-channel enhancement with $e^{\star}$ production in $e^{+} e^{-}$collisions. The differential cross-section is forward/backward peaked, an effect which is more pronounced for the lightest ordinary fermions; this mass effect however disappears when we keep to scattering angles $|\cos \theta| \leq 0.8$. In terms of $a=m_{f^{*}}^{2} / s$, the total cross section for $f^{*}$ production is [15]

$\sigma=\sigma_{0} N_{c} e_{f}^{4} f^{2} \frac{6 s}{\Lambda^{2}}\left[a\left(2 a^{2}-2 a+1\right) \log \frac{(1-a)^{2}}{m_{f}^{2} / s}+\frac{a\left(1-a-2 a^{3}\right)}{1+a} \log a+(1-a)\left(3+4 a^{2}\right)\right]$

Even in the case where the particles are produced at small angles, which accounts for a large part of the cross section, the events are not lost since the decay products of the $f^{\star}$ are at large angles. As Fig. 10d shows, $\sigma$ increases with $m_{f \star}$ up to nearly the kinematical limit where it starts bending over. The importance of the $\gamma \gamma$ mode compared to $e^{+} e^{-}$is that single production (even for $d$-type quarks where the charge is penalizing, and for a cut $|\cos \theta| \leq 0.8)$ is larger for all flavours except for the $e^{*}$ with $m_{e^{\star}}<700 \mathrm{GeV}$. The signals are quite clean: for $q^{\star}$, one can rely 
on the dominant decay $q^{\star} \rightarrow q g$, where the very energetic $q$ and $g$ jets are emitted at large angles (two hard QCD jet-events can be eliminated by imposing the cut $|\cos \theta|<0.8)$ while for $e^{*}$ one can use the $e^{*} \rightarrow e \gamma$ mode. Therefore, one can probe excited fermion masses of the order of $800 \mathrm{GeV}$, for reasonable $\Lambda$ values.

\subsection{Production in $e P$ Collisions}

Due to the special couplings of the electron to the excited leptons of the first generation, one can have single production of $e^{*}$ through $t$-channel $\gamma$ and $Z$ exchange, and $\nu^{*}$ through $t$-channel $W$ exchange in $e P$ collisions. Excited quarks of the first generation can also be produced in the same way, however background problems make this possibility less interesting than the production of excited leptons to which we stick here [49]. Using the scaled mass $a=m_{f^{\star}}^{2} / \hat{s}$, the deep inelastic differential cross section for the process $e P \rightarrow l^{*} X$ reads 15

$$
\begin{gathered}
\frac{\sigma}{d x d y}=2 \pi \alpha^{2} \frac{\hat{s}^{2}}{\Lambda^{2}} y \sum_{q, \bar{q}}\left[A_{l}\left(Q^{2}\right) R(x, y) q\left(x, Q^{2}\right)+\bar{A}_{l}\left(Q^{2}\right) \bar{R}(x, y) \bar{q}\left(x, Q^{2}\right)\right] \\
\text { with } \quad R(x, y)=2-(2-a)(y+a), \quad \bar{R}(x, y)=a(2-y-a)
\end{gathered}
$$

and in terms of the quark couplings to the gauge bosons, $A_{l}$ and $\bar{A}_{l}$ are defined by

$$
\begin{gathered}
A_{\nu}=\bar{A}_{\nu}=\frac{f_{W}^{2}}{4 s_{W}^{2}} \frac{1}{\left(Q^{2}+M_{W}^{2}\right)^{2}} \\
A_{e}=\frac{e_{q}^{2} f_{\gamma}^{2}}{\left(Q^{2}\right)^{2}}+\frac{2 e_{q} v_{q} f_{\gamma} f_{Z}}{Q^{2}\left(Q^{2}+M_{Z}^{2}\right)}+\frac{\left(v_{q}^{2}+a_{q}^{2}\right) f_{Z}^{2}}{\left(Q^{2}+M_{Z}^{2}\right)^{2}} \quad \bar{A}_{e}=\frac{2 e_{q} a_{q} f_{\gamma} f_{Z}}{Q^{2}\left(Q^{2}+M_{Z}^{2}\right)}+\frac{2 v_{q} a_{q} f_{Z}^{2}}{\left(Q^{2}+M_{Z}^{2}\right)^{2}}(27)
\end{gathered}
$$

In addition to the previous contribution (with a $Q^{2}$ cut of $5 \mathrm{GeV}^{2}$ ), one has two other contributions for the $e^{*}$ : one due to low $Q^{2}$ deep inelastic scattering and another due to the elastic process $e P \rightarrow e^{*} P$. The integrated total cross sections for $e^{*}$ and $\nu^{*}$ production are shown in Fig. 11a for $\Lambda / f=1 \mathrm{TeV}$. For $e^{*}$ the three different contributions discussed above are shown separately. Due to the low $Q^{2} t$ channel photon exchange, the $e^{*}$ total cross section is about an order of magnitude larger than for $\nu^{*}$. A clean detection channel will be provided by the wide angle electron-photon pair final state in the case of the $e^{*}$, and the electron- $W$ final state in the case of the $\nu^{*}$. Requiring 20 such events and assuming an integrated luminosity of $1 \mathrm{fb}^{-1}$, masses up to $800 \mathrm{GeV}$ for $\nu^{*}$ and $e^{*}$ can be probed.

The excited leptons (for $e^{*}$ those produced in the deep inelastic process) have larger $P_{T}$ than exotic leptons; Fig. 11b. This feature, in addition to the different $y$ distributions, can help to disentangle between the two sorts of new leptons which have the same decay modes and branching ratios (for the new electrons the distinction can easily be made because the $e^{\star}$ can decay into a photon). An additional way to disentangle between the two different sorts of neutral heavy leptons, is the completely different final polarization as shown in Fig. 11c for the $l^{*}$ (it is almost the same for $e^{*}$ and $\nu_{e}^{*}$. 
Figure 11: Total cross sections for the single production of first generation excited leptons in $e P$ collisions with $\sqrt{s}=1.2 \mathrm{TeV}$ (a); the transverse momentum distribution (b) and the longitudinal and transverse polarizations (c).

\subsection{Production in $p p$ Collisions}

Excited quarks can be produced in $p p$ collisions through a variety of mechanisms [50, 39]. The dominant production channels are the gluonic excitation of quarks $g+q \rightarrow q^{*}$ which occurs through the $q^{*} q g$ "gauge" interaction described previously, and the excitation through preon interactions $q q \rightarrow q q^{*}$ and $q^{*} q^{*}$; through contact interactions excited leptons, too, could eventually be produced at observable rates, $q \bar{q} \rightarrow e e^{*}$ and $e^{*} e^{*}$. The signatures of excited quarks are bumps in the invariant energies of jets, jet + gauge boson and jet + lepton pair combinations. Excited leptons would reveal themselves in bumps of leptons, leptons + gauge particles or leptons + quark jets. The first indication for the production of novel excited fermions could be the copious production of leptons, at large rates unexpected in the framework of the Standard Model.

The cross section for the gluonic excitation of quarks $g q \rightarrow q^{*}$ in $p p$ colliders is given by (we have taken $\Lambda=m_{*}$ in the numerical analysis) 50

$$
\sigma=\frac{\alpha_{s} \pi^{2}}{3 \Lambda^{2}} \tau \frac{d \mathcal{L}^{g q}}{d \tau} \quad, \quad \tau=\frac{m_{*}^{2}}{s}
$$

where $d \mathcal{L}^{g q} / d \tau$ is the quark-gluon luminosity for the $p p$ beams. The production cross section is shown by the full line in Fig. 12a, for the LHC at $\sqrt{s}=14 \mathrm{TeV}$. Given an integrated luminosity of $10 \mathrm{fb}^{-1}$, a mass range of $5-6 \mathrm{TeV}$ can be reached in this channel, based on 100 to 1000 events. The signals for singly produced excited quarks are large transverse momentum $j j, j \gamma, j Z$ or $j W$ pairs peaking at the mass of the resonance. Because the final states of the signal consist of large $P_{T}$ jets with large angles $\theta_{j j}$ between the jets of each pair, we introduced the following cuts to reduce the background:

$$
\theta_{j j}>30^{\circ}, \quad P_{T}>100 \mathrm{GeV}, \eta<2.5, \quad E^{t r}>m_{*} / 2
$$

The mass resolution is determined by the decay width of the resonance and the experimental jet resolution, which is taken to be $\Delta E / E=0.35 / \sqrt{E}+0.02$.

Excited quarks of the first generation can also be produced via contact interactions (which for large masses can overwhelm the gauge interactions) in the processes $q q \rightarrow q q^{*}$ and $q q \rightarrow q^{*} q^{*}$; for the value $\Lambda=m_{*}$ one can reach $q^{\star}$ masses of the order of $6 \mathrm{TeV}$ and $4 \mathrm{TeV}$ respectively; Fig. 12b. The backgrounds, which together with the cross sections have been calculated in [50, are well under control as shown in the figure. Through contact interactions, excited leptons could also be produced copiously in the processes $q \bar{q} \rightarrow e e^{*}$ and $q \bar{q} \rightarrow e^{*} e^{*}$. The cross sections, which are shown in Fig. 12c, are large and the signals, consisting of pure leptonic channels, would provide very clear signatures for the experimental identification of these novel states [the backgrounds are very rare in the SM]. For a luminosity of $10 \mathrm{fb}^{-1} e^{*}$ masses up to $\sim 4 \mathrm{TeV}$ could be accessible for $\Lambda=m_{*}$ [39]. 
Figure 12: Total cross sections for the production of (first generation) excited quarks through gauge interactions (a) or contact interactions (b) and of excited leptons through contact interactions in $p p$ collisions with $\sqrt{s}=14 \mathrm{TeV}$.

\section{Difermions}

\subsection{Leptoquark Production at $e^{+} e^{-}$Colliders}

There is much interest in the study of leptoquarks (LQ), colour (anti-)triplet, spin0 or spin-1 particles, which carry both baryon and lepton quantum numbers. As discussed in the introduction, such objects appear in a large number of extensions of the SM such as GUT's, Technicolour, and composite models. Quite generally, the signature for leptoquarks is striking: a high $p_{t}$ lepton balanced by a jet, or missing $p_{t}$ balanced by a jet, for the $\nu q$ decay mode, if applicable.

Single and pair production of scalar LQ's at a linear $e^{+} e^{-}$collider of $\sqrt{s}=1 \mathrm{TeV}$ was summarized by [51]. All three modes of the collider $-e^{+} e^{-}, e \gamma$ and $\gamma \gamma-$ were analyzed. We consider first the quark-level contribution to the process $e^{-} \gamma \rightarrow q S$, where $S$ is the LQ [51]. (This process was first considered by [52].) We parameterize the strength of the LQ coupling by comparing it to the electromagnetic interaction, i.e., $g_{L Q}^{2}=4 \pi k \alpha_{e m}$, and allow $k$ to vary. The cross section is

$$
\sigma(s)=\frac{\pi k \alpha_{e m}^{2}}{2 s}\left(Q_{S}+1\right)^{2}\left(1-2 \alpha+2 \alpha^{2}\right) \ln \left[\frac{s}{4 m_{q}^{2}}(\alpha+\beta)^{2}\right]+\ldots
$$

where '...' indicates additional (subdominant) terms, $Q_{S}$ is the LQ charge and

$$
\alpha \equiv 1-\left(M_{S}^{2}-m_{q}^{2}\right) / s, \quad \beta^{2} \equiv 1-2\left(M_{S}^{2}+m_{q}^{2}\right) / s+\left(M_{S}^{2}-m_{q}^{2}\right)^{2} / s^{2}
$$

Note that, due to the factor $\left(Q_{S}+1\right)^{2}$ in eq. 29, the production cross sections for LQ's of charge $-5 / 3$ and $-1 / 3$ are equal, as are those for $Q_{S}=-4 / 3$ and $-2 / 3$ (up to subdominant terms). The apparent divergence in the case of massless quarks is removed when detector cuts are imposed.

There are additional contributions to LQ production due to the hadronic content of the photon [53]. These can be taken into account by using a resolved photon, i.e. a photon distribution function. In Fig. 13 we compare the two contributions for $\sqrt{s}_{e e}=1 \mathrm{TeV}$, for $Q_{S}=-5 / 3$ and $k=1$, using the GRV distribution functions 54 with $Q^{2}=M_{S}^{2}$. In this figure we have folded in the photon energy spectrum due to the backscattered laser light. One sees that the resolved photon contribution is larger than the direct contribution for all LQ masses. This is easily understood physically: when one uses a resolved photon, one is actually considering the process $e^{-} \gamma \rightarrow S X$, where $X$ is not simply $q$ (as was the case above), but rather includes all sorts of soft hadronic products. Since more final states are included, relative to the direct process, it is only natural that the cross section should be larger. It 
should be pointed out, however, that, except for very light LQ's, the enhancement is only a factor of 2-3. In what follows we will consider only the resolved photon contributions to the different processes.

The dominant contribution to single LQ production in $e^{+} e^{-}$collisions comes from the sub-process $e^{-} \gamma \rightarrow S q$, in which the photon is radiated from the $e^{+}$. The cross section for $e^{+} e^{-} \rightarrow e^{+} S q$ is then calculated using the effective photon approximation. In addition, an $e^{+} e^{-}$collider can be turned into an $e \gamma$ or $\gamma \gamma$ collider by backscattering laser light from one or both of the beams. In calculating cross

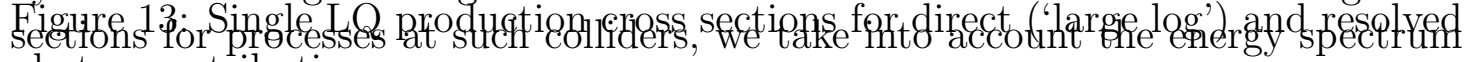
Bhotien backtributetioz's photons.

In Fig. 14 we compare the single-LQ production cross sections for all LQ charges, for $k=1$, at $e^{+} e^{-}$, e $\gamma$ and $\gamma \gamma$ colliders. There are several features to these figures. First, just as was the case for the direct contributions, the cross sections for LQ's of charge $-5 / 3$ and $-1 / 3$ are equal, and similarly for $Q_{S}=-4 / 3$ and $-2 / 3$. Second, at $e^{+} e^{-}$and $e \gamma$ colliders, only those LQ's which couple to the first generation (eu or ed LQ's) can be produced, but LQ's of all three generations can be produced at $\gamma \gamma$ colliders. Note that, since the $t$-quark distribution function is unknown, for the third-generation LQ with $Q_{S}=-5 / 3$ we calculated the direct contribution only.

As a figure of merit, we assume a luminosity of $60 \mathrm{fb}^{-1}$, and require 25 events for discovery. This implies that a LQ is observable if its production cross section is larger than $0.4 \mathrm{fb}$. It is clear from Fig. 15 that the $e \gamma$ mode is the best way to look for LQ's. For all charges, LQ's of mass up to about $900 \mathrm{GeV}$ are observable. For $Q_{S}=-5 / 3$, one can go slightly beyond this limit in the $e^{+} e^{-}$mode. $\gamma \gamma$ colliders are clearly not competitive for first-generation LQ's. However, second- and thirdgeneration LQ's are visible for certain ranges of masses. For other coupling, one simply scales the curves linearly in $k$. Thus, at $e \gamma$ colliders, first-generation LQ's will be observable even for couplings as weak as $k \lesssim 10^{-2}-10^{-3}$.

For all three colliders, the bulk of the cross section comes from those processes in which all particles go directly down the beam pipe. However, the LQ will subsequently decay, and its decay products will be seen in the detector. The signal will therefore simply consist of a lepton and a jet with a negligible SM background. 


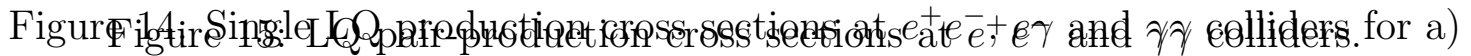
$Q_{S}=-5 / 3$ or $-1 / 3$, b) $Q_{S}=-4 / 3$ or $-2 / 3$.

For very weak LQ couplings, a better signal rate may be obtained from pair production of LQ's at $e^{+} e^{-}$or $\gamma \gamma$ colliders. Of course, the search is limited to LQ's of mass less than half of the c.m. energy of the collision. The cross sections for such a process are presented in Fig. 15. At $e^{+} e^{-}$colliders, there are two contributing diagrams, one of which depends on the LQ coupling $k$. The other diagram depends on the LQ's charge and weak isospin (in what follows, we assume $I_{3}=-1 / 2$ for LQ's with $Q_{S}=-5 / 3$ and $I_{3}=0$ for $\left.Q_{S}=-1 / 3\right)$.

At $\gamma \gamma$ colliders, on the other hand, the cross section is $k$-independent, depending solely on $Q_{S}$. In Fig. 15a, we show the pair-production cross sections for a LQ of charge $-5 / 3$. At $e^{+} e^{-}$colliders, we see that one loses about an order of magnitude in cross section as one passes from $k=1$ to $k=0$. However, regardless of the coupling, pair production of LQ's is observable for LQ masses essentially up to $\sqrt{s} / 2$. For comparison we also show the single-LQ production cross section. Both single- and pair-production cross sections are roughly of the same size for $k=1$, within the given LQ mass range, but the pair-production cross section wins out for smaller values of $k$. (Note that, in pair production, there may be more SM background). We also show the cross section at $\gamma \gamma$ colliders, including both the $\gamma \gamma$ - and $\gamma g$-initiated pair production. Unless $k$ is significantly bigger than 1 , the LQ pair-production rate is greater at $\gamma \gamma$ colliders than at $e^{+} e^{-}$colliders for the smaller values of the LQ mass. In Fig. 15b we present the cross sections for the smallest value of the LQ charge, $Q_{S}=-1 / 3$. Here we see that LQ pair production proceeds at a much greater rate at $e^{+} e^{-}$colliders, for $k=1$. For $k=0$, on the other hand, the $\gamma \gamma$ collider is better for smaller LQ masses. Again, LQ's of any generation are observable in this process for masses up to a bit less than $\sqrt{s} / 2$.

We conclude that it will be necessary to use all three modes of the linear $e^{+} e^{-}$ collider, and to consider both single and pair production, in order to perform a complete search for LQ's.

Although the discovery of a leptoquark would be dramatic evidence for physics beyond the SM, it would lead to the question of which model the leptoquark originated from. Given the large number of leptoquark types it would be imperative to measure its properties to answer this question. Following the notation of Ref. [19], the complete set of possible LQ are: $S_{1}, \tilde{S}_{1}$ (scalar, iso-singlet); $R_{2}, \tilde{R}_{2}$ (scalar, iso-doublet); $S_{3}$ (scalar, iso-triplet); $U_{1}, \tilde{U}_{1}$ (vector, iso-singlet); $V_{2}, \tilde{V}_{2}$ (vector, iso-doublet); $U_{3}$ (vector, iso-triplet). The production and corresponding decay signatures are quite similar, though not identical, and have been extensively studied. Even focussing only on the NLC ( $e^{+} e^{-}, e \gamma$ and $\gamma \gamma$ modes), there is a already a considerable amount of work in the literature [55]. The possibility of using a polarized $e \gamma$ collider to differentiate the LQ type (i.e., a polarized $e$ beam in conjunction with a polarized-laser backscattered photon beam) was analyzed here [56] including the contributions due to the hadronic content of the photon. An integrated luminosity of $50 \mathrm{fb}^{-1}$ was assumed. 
Figure 16: $A_{L L}$ vs. $M$ for (a) LQs which couple only to LH electrons for a $1 \mathrm{TeV}$ collider. The (solid, dashed, dotted, dot-dashed) curves are for $\left(S_{3}, \tilde{V}_{2}, U_{3}, \tilde{R}_{2}\right)$ LQs. (b) Same as (a) but for LQs which couple only to both LH and RH electrons; here $\kappa_{L}=1=\kappa_{R}$. The (solid, dashed, dotted, dot-dashed) curves are for $\left(S_{1}, V_{2}, U_{1}, R_{2}\right)$ LQs.

Table 2 of Ref. [19 gives information on the couplings to various quark and lepton combinations; note that both the quark and lepton have the same helicity (RR or LL) for scalar LQ production while they have opposite helicity (RL or LR) for vector LQ production. Denoting the various helicity cross sections as $\sigma^{\lambda_{e} \lambda_{q}}$, $\lambda_{i}=+$ for R helicity, $\lambda_{i}=-$ for L helicity and $\sigma_{T O T}=\sigma^{++}+\sigma^{+-}+\sigma^{-+}+\sigma^{--}$, it is useful to introduce the double longitudinal spin asymmetry $A_{L L}$ :

$$
A_{L L}=\frac{\left(\sigma^{++}+\sigma^{--}\right)-\left(\sigma^{+-}+\sigma^{-+}\right)}{\left(\sigma^{++}+\sigma^{--}\right)+\left(\sigma^{+-}+\sigma^{-+}\right)}
$$

which can be calculated for each LQ species. $100 \%$ polarization of both beams is assumed. In the analysis [56], the asymptotic polarized photon distribution functions [57] were used, and it is assumed that $Q^{2}$ and $x$ are large enough that the Vector Meson Dominance part of the photon structure is not important, but rather the behavior is dominated by the point-like $\gamma q \bar{q}$ coupling.

LQ's were identified only in the $e j$ mode. LQ's of spin-0(1) were found to have positive(negative) values of $A_{L L}$; for LQ's which couple in a $\mathrm{RH}$ manner to $e$ 's, this is sufficient to separate all cases. For the case of a $1 \mathrm{TeV}$ collider, Figs. 16ab show $A_{L L}$ as a function of the LQ mass including statistical errors for several LQ species. When LH couplings are present, then the values of $A_{L L}$ must be carefully examined. With statistical errors only, an NLC working in the $\gamma e$ mode could separate all LQ types up to approximately $80 \%$ of the center of mass energy assuming LQ Yukawa couplings of order electromagnetic strength. The largest uncertainty in the calculation is the reliability of the asymptotic approximation used to determine the photon distribution functions and those associated with the quark content of polarized photons.

The effects of QED and QCD corrections on the production of both vector (V) and scalar (S) LQ pairs in $e^{+} e^{-}$collisions at the NLC were considered [58]. These corrections were found to be critical, if one is to differentiate the different LQ species, since they significantly modify total cross sections and asymmetries. The vector LQ's were assumed to have minimal gauge boson couplings in this analysis so that the possibility that vector LQ are gauge particles was not covered. Initial state QED corrections were performed using the structure function approach including terms up to order $\alpha^{2}$ with soft photons exponentiation. Beamstrahlung corrections were also taken into account. The full set of QED corrections were found to be only weakly sensitive to variations in $\sqrt{s}$ in the range $0.5-1 \mathrm{TeV}$. However, bremsstrahlung corrections were found to be quite important in the threshold 
region, being of order $30-50 \%$ depending on the LQ spin.

For the scalar case, the QCD final state corrections were large and positive increasing dramatically near threshold indicating possible bound state formation. The QCD corrections were always in excess of $40 \%$ and determined to be more than $100 \%$ for LQ velocities below 0.2 due to the possible production of bound states and the well known Coulomb singularity.

Figure 17: 95\% C.L. discovery region (to the left of the curves) in the leptoquark coupling-mass plane for (a) LEP II (200 GeV) and an integrated luminosity of $100,200,500 \mathrm{pb}^{-1}$ corresponding to the dashed, dash-dotted, and solid curves, respectively. (b) NLC with a center of mass energy of $0.5(1.0) \mathrm{TeV}$ and $50(100) \mathrm{pb}^{-1}$, corresponding to the solid (dashed) curves, respectively.

Leptoquarks can participate in the process $e^{+} e^{-} \rightarrow q \bar{q}$ via virtual $u$ - or $t$ channel exchanges and can produce deviations from the SM predictions for cross sections and asymmetries [59]; thus indirect limits on LQ properties can be obtained. If one allows for the general form of leptoquark-fermion interactions of $\mathcal{L}=\ell\left(A+B \gamma_{5}\right) q \cdot \mathrm{LQ}$, the leptoquark couplings can be parameterized in terms of two constants, $\kappa \equiv\left(|A|^{2}+|B|^{2}\right) / e^{2}$ and $\kappa^{\prime}=2 \mathcal{R} e\left(A^{*} B / e^{2}\right)$. Here we examine the case $\kappa^{\prime}=\kappa$ and limit our discussion to the exchange of charge $-1 / 3$ leptoquarks present in $E_{6}$ theories, which mediate the reactions $e^{+} e^{-} \rightarrow u \bar{u}, c \bar{c}, t \bar{t}$.

The results are not found to be qualitatively different for $Q=+2 / 3$ leptoquark exchange or for considering the other extreme case of the parameters $\kappa^{\prime}=-\kappa$. The $95 \%$ C.L. discovery reach in the leptoquark coupling-mass plane is presented in Fig. 17 for LEP II with $\sqrt{s}=200 \mathrm{GeV}$, and the NLC for $\sqrt{s}=0.5,1.0 \mathrm{TeV}$. These search regions are obtained via a combined analysis of production cross sections, forward-backward asymmetries, and left-right polarization asymmetries (for the NLC only), assuming a beam polarization of $90 \%$ and a $50 \%$ efficiency for the identification of final state charm particles. Clearly, this process offers a good tool in the exploration of indirect leptoquark effects.

\subsection{Leptoquark Production at Hadron Colliders}

The calculations for the production of scalar and vector LQ's at hadron colliders, both singly and in pairs has been updated [60] for this report. The results in the scalar case were obtained long ago [61, so we briefly discuss the intricacies of the vector case below.

In order to determine the $q \bar{q}, g g \rightarrow V V$ cross sections we need to determine both the trilinear $g V V$ and quartic $g g V V$ couplings, which may naively at first appear to be unknown. However, in any realistic model wherein vector leptoquarks appear and are fundamental objects, they will be the gauge bosons of an extended gauge group. In this case the $g V V$ and $g g V V$ couplings are completely fixed by

gauge invariance. These particular couplings will also insure that the subprocess cross section obeys tree-level unitarity, as is the hallmark of all gauge theories. Of 
course, it might be that the appearance of vector leptoquarks is simply some low energy manifestation of a more fundamental theory at a higher scale and that these particles may even be composite, in which case so-called 'anomalous' couplings in both the $g V V$ and $g g V V$ vertices can appear. One such possible coupling is an 'anomalous magnetic moment', usually described in the literature by the parameter $\kappa$ [62], which takes the value of unity in the gauge theory case.

Figure 18: Production cross section for a pair of vector leptoquarks at the Tevatron: (a) as a function of the LQ mass with $\kappa=1$. The dotted(dashed, solid)curve corresponds to the $q \bar{q}(g g$, total) contribution. The dash-dotted curve is the total S-LQ result. (b $\kappa$ dependence of the $q \bar{q}$ (dots), $g g$ (dashes), and total(solid) $V$ pair production cross sections at the Tevatron for a vector leptoquark mass of $200 \mathrm{GeV}$.

Figure 19: Same as the previous figure but for the LHC. In (b), a vector leptoquark mass of $1 \mathrm{TeV}$ is assumed.

Among these 'anomalous couplings', the term which induces $\kappa$ is special in that it is the only one that conserves $C P$ and is of dimension 4. As values of $\kappa$ differing from one have been entertained in the literature, we will generally assume $\kappa=1$ or 0 , with the latter value corresponding to 'minimal' coupling, in order to probe the sensitivity of our results to the assumed gauge nature of $V$. We will also describe the results in the more general case where $\kappa$ is arbitrary.

If LQ's are first observed at hadron colliders it will become necessary to be able identify which one [19 has been found. The simplest probe of LQ properties is the cross section itself. The two individual subprocess result in the total cross sections displayed in Figs. 18a-b and 19a-b at the Tevatron and LHC and are compared with the scalar LQ case. As we see from these figures, the production rate for spin-1 LQs can be substantially larger than in the spin-0 case and there exists a reasonable sensitivity to the choice of $\kappa$. At the LHC with $100 \mathrm{fb}^{-1}$ the search reach for scalar(S)/vector(V) LQ's is $1.4 / 2.2(1.8) \mathrm{TeV}$ for $\kappa=1(0)$. At the Tevatron with 200(2000) $\mathrm{pb}^{-1}$, the V-LQ reaches are $300(385) \mathrm{GeV}$ for $\kappa=1$ and 250(330) GeV for $\kappa=0$. The corresponding results for S-LQ limits are 170(250). At a $4 \mathrm{TeV} p \bar{p}$ collider with $1 \mathrm{fb}^{-1}$ the $\mathrm{V}$-LQ reach is $850 \mathrm{GeV}$ with $\kappa=1$, while for scalars it is $620 \mathrm{GeV}$. Correspondingly, at a $100 \mathrm{TeV} p p$ collider with $100 \mathrm{fb}^{-1}$ the V-LQ limit is $8.2 \mathrm{TeV}$ with $\kappa=1$, while for S-LQ's it is $5.0 \mathrm{TeV}$. All these results assume a branching fraction of unity for the $e j$ final state. 
Single LQ production has the advantage of a larger available phase space but has the disadvantage associated with the fact that cross sections are proportional to an unknown Yukawa coupling, which is expected to be order electroweak strength or less. In the V-LQ case, the arbitrariness of $\kappa$ still enters the calculation and care must be taken to distinguish possible $g u$ from $g d$ production. (For example, the $E_{6}$ type LQ with $Q=-1 / 3$ is produced via $g u \rightarrow L Q+e^{+}$and $g d \rightarrow L Q+\bar{\nu}$ with different Yukawa couplings.) Figs. 20 and 21 show the single LQ rates for both the LHC and Tevatron for either production process; $\kappa=0,1$ are considered in the V-LQ case. It is assumed that all Yukawa couplings are exactly equal to $\alpha$ in these figures. Clearly, if the Yukawa's are significantly smaller pair production will generally win out over single production for the entire mass range.

Figure 20: Single (a)S-LQ (b)V-LQ production at the Tevatron for unit scaled Yukawa coupling. In (a) the dotted(dashdotted) curve is for $g u(g d)$ production. In (b), the upper(lower) pair of curves corresponds to $g u(g d)$ production with $\kappa=1,0$ respectively.

Figure 21: Same as the previous figure but for the LHC.

There are other mechanisms that produce LQ's at hadron colliders. In an alternative supersymmetric version of the LRM [63], which takes advantage of a well known ambiguity in the fermion quantum number assignments within the $\mathbf{2 7}$ representation of $E_{6}, W_{R}$ 's can only be produced in association with leptoquarks(LQ) at hadron colliders. The basic process is $g u \rightarrow W_{R}+L Q$. The discovery limit of order $1.2-2.5 \mathrm{TeV}$ is obtained in the background-free case at the LHC for an integrated luminosity of $100 \mathrm{fb}^{-1}$. 
A detailed study has been performed for this report [64], in order to ascertain the extent to which the backgrounds from top and SM $W$ boson leptonic decays masked the signal at the LHC. It was assumed that the final state neutral lepton appears as missing energy and that the signals and backgrounds are well modeled via PYTHIA.

If one completely ignores the associated LQ, the signal corresponds to an increase in the overall lepton $p_{t}$ distribution in the region corresponding to the $W_{R}$ 's Jacobian peak. For $p_{t}>M_{W_{R}}$, the signal surpasses the backgrounds from $t \bar{t}$ production with rates of order 10-100 events a year assuming $M_{W_{R}}=1 \mathrm{TeV}$ and $\Delta p_{t}=100 \mathrm{GeV}$. However, this signal is overwhelmed by the inclusive lepton spectrum from the decay of the SM $W$ boson (due to the fact that at large $p_{t}$ associated $W_{L}+j$ dominates) by an order of magnitude.

This situation was found to be somewhat improved if the leptonic decay modes of the LQ were included, i.e., $L Q \rightarrow u e+\chi^{0}$, de $+\chi^{+}$, where $\chi^{+}\left(\chi^{0}\right)$ is a chargino (neutralino or LSP). The signal now corresponds to a charged lepton pair plus a jet plus missing energy. Unfortunately, although the background from $W_{L}$ is now removed, that from $t \bar{t}$ still remains and swamps the signal by two orders of magnitude for a $1 \mathrm{TeV} W_{R}$ and typical SUSY partner masses.

The conclusion of the analysis is that this final state is not suitable for $W_{R}$ discovery for small LQ masses (i.e., below those of the SUSY particles) due to the tiny leptonic branching fraction possessed by the LQ in this case. 


\subsection{Single Dilepton Production at $e^{+} e^{-}$Colliders}

Dileptons arise in theories where the gauge group for leptons is expanded from the $\mathrm{SU}(2)_{L}$ of the $\mathrm{SM}$ to $\mathrm{SU}(3)$. They can appear as both scalars and as vector gauge particles, and can be singly- or doubly-charged. The production of doubly-charged scalar and vector dileptons in $e^{+} e^{-}, \gamma \gamma$ and $e \gamma$ colliders is summarized for this report [65]; for original references, see [21, 66].

Dilepton interactions are described by the Lagrangian

$$
\mathcal{L}_{\text {int }}=-\frac{g_{3 l}}{\sqrt{2}} X_{\mu}^{++} e^{T} C \gamma^{\mu} \gamma_{5} e+\frac{g}{\sqrt{2}} X^{++} e^{T} C\left(1-\lambda \gamma_{5}\right) e+h . c .
$$

where $X_{\mu}^{++}\left(X^{++}\right)$is the vector (scalar) dilepton field, and $C$ is the charge conjugation matrix. The vector coupling of the vector dilepton vanishes by Fermi statistics. The coupling of the scalar dilepton is chiral, so that $\lambda= \pm 1$. The coupling constants $g$ and $g_{3 l}$ are considered as free parameters.

The cross sections for the processes $e^{-} \gamma \rightarrow X_{S, V}^{--} e^{+}$, using $g_{3 l}^{2} \equiv 4 \pi k_{V} \alpha_{e m}$ and $g^{2} \equiv 4 \pi k_{S} \alpha_{e m}$ is given by ( $\alpha$ and $\beta$ are given in eq. 30 ).

$$
\begin{aligned}
\sigma_{S}(s)=\frac{\pi k_{S} \alpha_{e m}^{2}}{s}[\beta & \left(\frac{3}{2}+\frac{17}{2} \frac{M_{X}^{2}}{s}\right)+8 \frac{M_{X}^{2}}{s} \ln \left(\frac{2-\alpha-\beta}{2-\alpha+\beta}\right) \\
& \left.+\frac{s^{2}-2 s M_{X}^{2}+2 M_{X}^{4}}{s^{2}} \ln \left(\frac{\alpha+\beta}{\alpha-\beta}\right)\right] \\
\sigma_{V}(s)=\frac{\pi k_{V} \alpha_{e m}^{2}}{s}[\beta & \left(2+\frac{8 s}{M_{X}^{2}}+\frac{13}{2} \frac{M_{X}^{2}}{s}\right)+\frac{s^{2}-2 s M_{X}^{2}+2 M_{X}^{4}}{s^{2}} \ln \left(\frac{\alpha+\beta}{\alpha-\beta}\right) \\
& \left.+\frac{\left(-s^{3}+12 s^{2} M_{X}^{2}+18 s M_{X}^{4}-4 M_{X}^{6}\right)}{2 s^{2} M_{X}^{2}} \ln \left(\frac{2-\alpha-\beta}{2-\alpha+\beta}\right)\right]
\end{aligned}
$$

The explicit electron mass regulates the logarithmic collinear divergence occurring in that region of phase space in which all particles go down the beam pipe. (The apparent divergence in the massless electron limit can be removed by detector cuts since these will be present in any real cross section determination.) Thus, the bulk of the above cross sections are due to those events in which the only particles detected are the decay products of the $X_{S, V}^{--}\left(e^{-} e^{-}, \mu^{-} \mu^{-}\right.$or $\left.\tau^{-} \tau^{-}\right)$. This results in an unmistakable signature with virtually no SM background.

For the process $e^{+} e^{-} \rightarrow X_{S, V}^{--} e^{+} e^{+}$, an energetic virtual photon is emitted from the $e^{+}$beam, which then becomes part of an $e^{-} \gamma$ collision. To calculate this cross section, we use a photon distribution function for the virtual photon, and fold it together with the previously determined cross section of $e^{-} \gamma \rightarrow X_{S, V}^{-} e^{+}$, using numerical integration. The process $\gamma \gamma \rightarrow X_{S, V}^{--} e^{+} e^{+}$proceeds along similar lines as above, except that a virtual electron is emitted from the $\gamma$ beam, and a fermion distribution function is used.

In this summary, we show the production cross sections for vector and scalar dileptons in each process, at $1 \mathrm{TeV}$. Assuming a luminosity of $60 \mathrm{fb}^{-1}$, we suppose 
that 25 events, i.e. a cross section of $0.4 \mathrm{fb}$, are required for discovery. We take the coupling constants, $g_{3 l}$ and $g$, to be of electromagnetic strength, i.e. $k_{V}=1$ and $k_{S}=1$. Of course, since the cross sections are linear in $k_{V}$ and $k_{S}$, these graphs can easily be scaled to other parametric values of the couplings.

In Fig. 22a we see that the cross section for scalar dilepton production in $e \gamma$ collisions is huge, orders of magnitude above the discovery limit. Thus, scalar dileptons with masses virtually up to the kinematic limit are observable, even for couplings as small as $k_{S}=5-7 \times 10^{-4}$. The cross sections in $e^{+} e^{-}$and $\gamma \gamma$ collisions are smaller, but still large enough for the observation of dileptons with masses approaching the kinematic limit. Fig. 22b shows the production cross sections for vector dileptons. Once again, the cross section in the $e \gamma$ process is clearly much larger than that of the other two processes, so that dileptons of masses up to the kinematic limit and couplings as small as $k_{V}=3-4 \times 10^{-4}$ can be detected. Dileptons can be observed in $e^{+} e^{-}$and $\gamma \gamma$ collisions for $k_{V}$ as low as $\sim 0.01$.

This analysis shows that both scalar and vector dileptons can be easily observed in all three modes of the $e^{+} e^{-}$collider. The bulk of the cross sections comes from those events in which the only particles detected are the two leptons coming from the decay of the dilepton, an unmistakable signature.

Figure 22: Production cross section for (a) scalar (left) and (b) vector (right) dileptons in the processes $e^{-} \gamma \rightarrow X_{S}^{--} e^{+}$(solid line), $e^{-} e^{+} \rightarrow X_{S}^{--} e^{+} e^{+}$(dashed line) and $\gamma \gamma \rightarrow$ $X_{S}^{--} e^{+} e^{+}$(dash-dot line), for a $1 \mathrm{TeV}$ NLC with $k_{S}=1$. The horizontal line is the assumed discovery cross section of $0.4 \mathrm{fb}$.

\section{New Interactions}

\subsection{Top Quark Anomalous Chromomagnetic Moments}

The discovery of the top quark at the Tevatron [67] by the CDF and D0 Collaborations has renewed thinking about what may be learned from a detailed study of the properties of this particle. One point of view is that this discovery represents a great triumph and confirmation of the predictions of the SM, in that the top quark lies in the mass range anticipated by precision electroweak data 68. Another viewpoint is that the subtleties of top quark physics itself may shed some light on new physics beyond the Standard Model.

Amongst others, one set of the top quark properties which deserve study are its couplings to the various gauge bosons; up until recently such analyses 69 have concentrated on the electroweak couplings of the top, i.e., its interactions with the $W, Z$ and $\gamma$. In what follows, we consider the possible existence of an anomalous chromomagnetic moment, a dimension-5 coupling, $\kappa$, at the $t \bar{t} g$ 
vertex and we explore the capability of the Tevatron, LHC and NLC to probe this coupling. Such anomalous interactions may arise with a reasonable strength in extended technicolor or compositeness scenarios [70 and, e.g., may lead to significant alterations in the top production cross section at the Tevatron and other colliders. In such scenarios, the chromomagnetic moment is usually induced as a natural by-product of the top quark mass generation process. At the present time the CDF and D0 top cross section results seem to be in rough agreement with, although still somewhat larger than, the expectations of QCD [71]. The original version of this analysis was motivated by this somewhat larger than expected result first obtained by CDF last year. Previous to the present analysis, only rather weak limits on $\kappa$ (of order 10) existed, in particular, from operator mixing contributions to the $b \rightarrow s \gamma$ decay; see the last paper in 69]). Data from the Tevatron, LHC, and NLC will be able to improve this sensitivity by two orders of magnitude. For details of the analyses presented below, see ref. [72].

The piece of the Lagrangian which governs the $t \bar{t} g$ and $t \bar{t} g g$ couplings is:

$$
\mathcal{L}=g_{S} \bar{t} T_{a}\left(\gamma_{\mu} G_{a}^{\mu}+i \frac{\kappa}{2 m_{t}} \sigma_{\mu \nu} G_{a}^{\mu \nu}\right) t
$$

where $g_{S}$ and $T_{a}$ are the usual $S U(3)_{C}$ coupling and generators, $m_{t}$ is the top quark mass, $G_{a}^{\mu}\left(G_{a}^{\mu \nu}\right)$ is the gluon field (strength tensor), and $\kappa$ is the anomalous chromomagnetic moment which is zero in the SM. Note that $S U(3)_{C}$ gauge invariance requires that both vertices be modified when $\kappa$ is present.

Turning first to the examination of the effects of non-zero $\kappa$ on $t \bar{t}$ production at hadron colliders, we present the parton-level $q \bar{q} \rightarrow t \bar{t}$ and $g g \rightarrow t \bar{t}$ differential cross sections. (For single top production at these machines, we need the corresponding $g W \rightarrow t \bar{b}$ result.) We note in passing that the $q \bar{q}$ process dominates (about $90 \%$ ) at the Tevatron while the $g g$ one is similarly dominant at the LHC. This analysis has shown that the total top cross section is the most sensitive quantity in probing $\kappa$ at hadron colliders provided it is not too large. For $q \bar{q} \rightarrow t \bar{t}$, which occurs through $s$-channel gluon exchange, one obtains

$$
\frac{d \sigma_{q \bar{q}}}{d \hat{t}}=\frac{2 \pi \alpha_{S}^{2}}{27 \hat{s}^{2}}\left[\left(1+\frac{2 m_{t}^{2}}{\hat{s}}\right)+3 \kappa+\kappa^{2}\left(\frac{\hat{s}}{8 m_{t}^{2}}+1\right)+\frac{1}{4}\left(3 z^{2}-1\right)\left(1-\frac{\hat{s}}{4 m_{t}^{2}} \kappa^{2}\right)\right],
$$

with $\hat{s}$ being the parton level c.m. energy and $z$ the cosine of the corresponding scattering angle. The case of the $g g \rightarrow t \bar{t}$ is more complicated since it proceeds through $s^{-}, t-$, and $u$-channel diagrams as well as a contact term which is present due to gauge invariance; defining the kinematic abbreviations $x=m_{t}^{2} / \hat{s}, K=$ $\kappa /(2 \sqrt{x})$ and $d=1-z^{2}+4 x z^{2}$, the resulting differential cross section can be written as

$$
\frac{d \sigma_{g g}}{d \hat{t}}=\frac{\pi \alpha_{s}^{2}}{64 \hat{s}^{2}}\left[T_{0}+T_{1} K+T_{2} K^{2}+T_{3} K^{3}+T_{4} K^{4}\right]
$$

which is a quartic polynomial in $\kappa$, where the $T_{i}$ coefficients can be written as

$$
T_{0}=4\left(36 x z^{2}-7-9 z^{2}\right)\left(z^{4}-8 x z^{4}+16 x^{2} z^{4}-32 x^{2} z^{2}+8 x z^{2}-8 x-1\right) / 3 d^{2},
$$




$$
\begin{aligned}
& T_{1}=-32\left(36 x z^{2}-7-9 z^{2}\right) \sqrt{x} / 3 d \\
& T_{2}=-16\left(72 x^{2} z^{2}-46 x z^{2}+7 z^{2}-16 x-7\right) / 3 d \\
& T_{3}=32\left(-7 z^{2}+28 x z^{2}-5 x+7\right) \sqrt{x} / 3 d \\
& T_{4}=16\left(-8 x z^{4}+16 x^{2} z^{4}+z^{4}-4 x^{2} z^{2}+9 x z^{2}-2 z^{2}+1-x+4 x^{2}\right) / 3 d .
\end{aligned}
$$

Figure 23: (a) NLO cross sections for the $q \bar{q} \rightarrow t \bar{t}$ (dash-dotted) and $g g \rightarrow t \bar{t}$ (dotted) subprocesses as well as the total cross section (solid) at the Tevatron as functions of $\kappa$ for $m_{t}=170 \mathrm{GeV}$ using the CTEQ parton distribution functions. The horizontal dashed lines provide the $\pm 1 \sigma \mathrm{CDF}$ cross section determination while the horizontal dotted line is the D0 95\% CL upper limit. (b) Same as (a) but for the LHC and with the roles of $g g$ and $q \bar{q}$ interchanged.

Figure 24: Single top cross section at the (a) Tevatron and (b) LHC. From top to bottom, the curves correspond to $\kappa=2,-2,1,-1,0$.

As in the $q \bar{q}$ case, the $g g \rightarrow t \bar{t}$ cross section increases as $\kappa$ increases in the positive direction. These cross sections the for Tevatron and LHC are shown in Fig. 23a-b, respectively. If the SM cross section is realized, accounting for various uncertainties (parton densities, NNLO terms, scales, luminosity, statistics, etc.) the $95 \%$ CL ranges for $\kappa$ assuming Tevatron luminosities of $\mathcal{L}=100(250,500,1000)$ $\mathrm{pb}^{-1}$ are -0.14 to $0.15,-0.11$ to $0.12,-0.09$ to 0.11 , and -0.08 to 0.11 , respectively, i.e., they are systematics limited at large luminosity. The Tevatron analysis was then extended to the LHC case where it was found that the results were clearly systematics limited at the level of $\kappa \simeq \pm 0.10$ due to the uncertainties from higher order QCD corrections and parton density variations.

In the case of single production of top, we expect low sensitivity to $\kappa$ due to the dominance of the light $b$-quark exchange diagram. This is clearly the case as shown in Figs. 24a-b for the Tevatron and LHC.

At the NLC, the $t \bar{t} g$ vertex can only be directly explored via the QCD radiative process $e^{+} e^{-} \rightarrow t \bar{t} g$. Relative to the LHC and Tevatron, this results in a substantial loss in statistics which can be compensated for by the cleanliness of the environment as well as a reduction in the associated theoretical uncertainties. Since the new $\kappa$-dependent interaction is proportional to the gluon 4-momentum, one is lead to a study of the gluon energy distribution associated with $t \bar{t}$ production.

To leading order in $\alpha_{S}$ one can factorize this cross section into separate contributions due to the vector and axial-vector couplings of the top quark to the $s$-channel exchanged gauge bosons as

$$
d^{2} W / d z_{1} d z_{2}=F_{v} d^{2} W_{v} / d z_{1} d z_{2}+F_{a} d^{2} W_{a} / d z_{1} d z_{2}
$$

where $F_{v, a}$ are the 'weighting' factors. This result is scaled to the lowest order $t \bar{t}$ production cross section, i.e., $W=\sigma / \sigma_{0}$, where $\sigma_{0}=\sigma\left(e^{+} e^{-} \rightarrow t \bar{t}\right)$, and 


$$
F_{v}=\frac{\frac{1}{2} \beta\left(3-\beta^{2}\right) A_{v}}{\beta^{3} A_{a}+\frac{1}{2} \beta\left(3-\beta^{2}\right) A_{v}}, \quad F_{a}=\frac{\beta^{3} A_{a}}{\beta^{3} A_{a}+\frac{1}{2} \beta\left(3-\beta^{2}\right) A_{v}},
$$

with $\beta=\sqrt{\left(1-4 m_{t}^{2} / s\right)}$, and

$$
\begin{gathered}
A_{v}=\sum_{i j}\left(v_{i} v_{j}+a_{i} a_{j}\right)_{e}\left(v_{i} v_{j}\right)_{t} P_{i j}, \quad A_{a}=\sum_{i j}\left(v_{i} v_{j}+a_{i} a_{j}\right)_{e}\left(a_{i} a_{j}\right)_{t} P_{i j}, \\
P_{i j}=s^{2} \frac{\left[\left(s-M_{i}^{2}\right)\left(s-M_{j}^{2}\right)+(\Gamma M)_{i}(\Gamma M)_{j}\right]}{\left[\left(s-M_{i}^{2}\right)^{2}+(\Gamma M)_{i}^{2}\right]\left[\left(s-M_{j}^{2}\right)^{2}+(\Gamma M)_{j}^{2}\right]} .
\end{gathered}
$$

The sum in the expression above is over the $s$-channel $\gamma(i, j=1)$ and $Z(i, j=2)$ gauge boson exchanges. Defining the overall normalization coefficients

$$
N_{v}=\frac{2 \alpha_{s}}{3 \pi}\left(2 m_{t}^{2} s^{2} x_{1}^{2} x_{2}^{2}\right)^{-1}\left[\frac{1}{2} \beta\left(3-\beta^{2}\right)\right]^{-1}, \quad N_{a}=\frac{2 \alpha_{s}}{3 \pi}\left(2 m_{t}^{2} s^{2} x_{1}^{2} x_{2}^{2}\right)^{-1}\left[\beta^{3}\right]^{-1},
$$

where $x_{i}=1-z_{i}$, we obtain the complete $t \bar{t} g$ double differential cross section:

$$
\begin{aligned}
\frac{d^{2} W_{v}}{d z_{1} d z_{2}}= & N_{v}\left[-8 m_{t}^{6}\left(x_{1}+x_{2}\right)^{2}-4 s m_{t}^{4}\left[x_{1}^{2}\left(1+2 x_{2}\right)+x_{2}^{2}\left(1+2 x_{1}\right)\right]\right. \\
& +2 s^{2} m_{t}^{2} x_{1} x_{2}\left[\left(1-x_{1}\right)^{2}+\left(1-x_{2}\right)^{2}+\kappa\left(x_{1}-x_{2}\right)^{2}\right] \\
& \left.+\kappa^{2} s^{3} x_{1}^{2} x_{2}^{2}\left(1-x_{1}-x_{2}\right)\right], \\
\frac{d^{2} W_{a}}{d z_{1} d z_{2}}= & N_{a}\left[16 m_{t}^{6}\left(x_{1}+x_{2}\right)^{2}+2 s m_{t}^{4}\left[\left(\kappa^{2}+2 \kappa+2\right) x_{1} x_{2}\left(x_{1}+x_{2}\right)^{2}\right.\right. \\
& \left.+8 x_{1} x_{2}\left(x_{1}+x_{2}\right)-2\left(x_{1}^{2}+x_{2}^{2}+6 x_{1} x_{2}\right)\right]+2 m_{t}^{2} s^{2} x_{1} x_{2}\left[\left(1-x_{1}\right)^{2}\right. \\
& \left.+\left(1-x_{2}\right)^{2}+\kappa\left(x_{1}^{2}+x_{2}^{2}-4\right)+\kappa^{2} x_{1} x_{2}\left(x_{1}+x_{2}-3\right)\right] \\
& \left.+\kappa^{2} s^{3} x_{1}^{2} x_{2}^{2}\left(1-x_{1}\right)\left(1-x_{2}\right)\right]
\end{aligned}
$$

The dominant effect of $\kappa \neq 0$ is to induce an increase in the high energy tail of this distribution. This same energy dependence leads to the observation that the finite $\kappa$ contributions grow rapidly with increasing $\sqrt{s} / 2 m_{t}$, implying increased sensitivity at an NLC with $\sqrt{s}=1 \mathrm{TeV}$ instead of $500 \mathrm{GeV}$. In this first study, we ignore effects from top decay (except in the statistics) and perform a LO analysis. Estimates of contributions from higher order are lumped into the uncertainties when obtaining limits. Fig. 25 shows this distribution for the case of $\sqrt{s}=1$ $\mathrm{TeV}$ for $\alpha_{S}=0.10$ while Fig. 26a shows the result of integrating this distribution for values of $z=2 E_{\text {gluon }} / \sqrt{s}>0.4$. Assuming that the SM results are realized, bounds on $\kappa$ may be obtainable by either $(i)$ counting excess events with high energy gluon jets or $(i i)$ by a fit to the gluon energy distribution via a Monte Carlo analysis. Events are selected with at least one b-tag as well as one high $p_{t}$ lepton and gluon jet energies larger than $200 \mathrm{GeV}$. Such large jet energies will allow a 
clean separation from the top decays and will simultaneously place us in the region of greatest $\kappa$ sensitivity. For a luminosity of $200 \mathrm{fb}^{-1}$ the resulting $95 \%$ CL allowed range is found to be $-1.0 \leq \kappa \leq 0.25$. Substantial improvement is obtained by fitting the spectrum itself; Fig. 26b shows the Monte Carlo generated spectrum and best fit $(\kappa=0.06)$ assuming that the SM is realized. At $95 \% \mathrm{CL}$, one now obtains the allowed range of $-0.12 \leq \kappa \leq 0.21$ for the same luminosity as above.

The LHC, Tevatron and NLC provide complementary windows on the possible anomalous chromomagnetic couplings of the top with different systematics.

Figure 25: Gluon jet energy spectrum assuming $\alpha_{s}=0.10$ for $m_{t}=175 \mathrm{GeV}$ at a $1 \mathrm{TeV}$ NLC. The upper(lower) dotted, dashed, and dot-dashed curves correspond to $\kappa$ values of $3(-3), 2(-2)$, and 1(-1) respectively while the solid curve is conventional QCD with $\kappa=0$.

Figure 26: (a)Integrated gluon energy spectrum for the same input parameters and labeling as in the previous figure as a function of $\kappa$ assuming $z_{\text {cut }}=0.4$. (b)Best fit gluon spectrum through the points generated by the Monte Carlo analysis for $\kappa=0.06$.

\subsection{Top quark radius and anomalous magnetic moment}

One of the most natural consequences of substructure in the fermionic sector is the appearance of an intrinsic finite size of quarks and leptons due to the interaction of the preons, the more elementary constituents [73]. The search for non-zero radii and anomalous magnetic moments has been pursuit since a long time and stringent bounds on the size of electrons and muons have been set by measurements of $(g-2)_{e, \mu}$ 47. Any non point-like structure of these particles is restricted to energy scales above $1 \mathrm{TeV}$. Similar limits can be obtained for light quarks from analyses of quark-quark scattering in $p \bar{p}$ colliders $\| 74$ and for $\tau$ leptons and $b$ quarks from the high-precision LEP measurements [75].

These bounds for the light fermions cannot be readily extrapolated to the heavy top quark. Indeed, as its large mass seems to indicate, the top quark could play a special rôle and it may be the first place where non-standard effects will appear. (In models where the standard gauge symmetry is dynamically broken by $\bar{t}$ condensates, anomalous couplings are also expected to occur [76].) Here, we discuss the potential of a $0.5 \mathrm{TeV} e^{+} e^{-}$collider in probing anomalous couplings of the top quark to electroweak gauge bosons.

The general coupling of a gauge boson $\alpha=\gamma, Z$ to fermions can be written as

$$
i e_{0}\left[f_{1}^{\alpha} \gamma_{\mu}+\frac{i}{2 m_{f}} f_{2}^{\alpha} \sigma_{\mu \nu} q^{\nu}+f_{3}^{\alpha} \gamma_{\mu} \gamma_{5}+\frac{i}{2 m_{f}} f_{4}^{\alpha} \sigma_{\mu \nu} \gamma_{5} q^{\nu}\right]
$$

with $e_{0}$ being the proton charge and $q$ the momentum carried by the gauge boson. In principle, there are also $q^{\mu}$ and $q^{\mu} \gamma_{5}$ couplings, but they give vanishing contributions if the gauge boson is on shell or couples to massless fermions. In the point-like limit, the form factors $f_{1}-f_{4}$ reduce to the usual standard model 
couplings: the $\mathrm{CP}$ violating terms $f_{4}^{\gamma, Z}$ are absent and at the tree level $f_{2}^{\gamma, Z}$ are equal to zero, while $f_{1}^{\gamma}=e_{f}, f_{3}^{\gamma}=0$ and $f_{1}^{Z}=v_{f}^{Z}, f_{3}^{Z}=a_{f}^{Z}$. The form factors $f_{1,3}$ are related to the radius $R$ which is proportional to the compositeness scale $R=\sqrt{6 / \Lambda}: f_{1,3}^{\alpha} \sim\left(f_{1,3}^{\alpha}\right)^{S M}\left(1+s / \Lambda^{2}\right)$; they could in principle be different for $\gamma$ and $Z$ couplings. Note that the radius used here is the physical particle radius which is not plagued by the ambiguities due to the unknown coupling constants as it is the case in contact terms. $f_{2}^{\gamma, Z}$ are the anomalous magnetic moments which, in chiral theories as suggested by $(g-2)_{e, \mu}$, are proportional to $m_{f}^{2} / \Lambda^{2}$.

With the general form given above, one can write the most general expression for the differential cross section $\mathrm{d} \sigma / \mathrm{d} \cos \theta$ in the processes $e^{+} e^{-} \rightarrow f \bar{f}$ and $\gamma \gamma \rightarrow f \bar{f}$ [77]. In $e^{+} e^{-}$annihilation, this cross section allows three independent measurements and one may choose $R_{f}$ the total cross section normalized to $\sigma_{0}$, the forward-backward asymmetry $A_{f}$ of the fermion and the parameter $\alpha_{f}$ defined by $\mathrm{d} \sigma / \mathrm{d} \cos \theta \propto 1+\alpha_{f} \cos ^{2} \theta$; the expressions are given in [77]. These observables have to be compared to the experimental data once the radiative corrections of the SM have been properly taken into account. At a $0.5 \mathrm{TeV} e^{+} e^{-}$collider and for $m_{t}=175 \mathrm{GeV}$, we show in Fig. 27 the deviations of the three observables from the SM expectations as a function of the compositeness scale $\Lambda$. We have set the CP Figure 27 Deviations of the normalized cross section $R_{t}$, the forward-backward asymmetry $A_{t}$ and the $\alpha_{t}$ parameter from SM expectations in $e^{+} e^{-} \rightarrow t \bar{t}$ at $\sqrt{s}=0.5 \mathrm{TeV}$, for different values of the form-factors $f_{i}$.

Fig. 27a shows the deviations in the case where $\delta f_{1,3}^{Z}=\delta f_{1}^{\gamma}=s / \Lambda^{2}$ and $f_{2}^{Z}=$ $f_{2}^{\gamma}=m_{t}^{2} / \Lambda^{2}$. The shifts can be very large especially for relatively small values of $\Lambda$. The ratio $R_{t}$ is the most sensitive quantity and an experimental accuracy of $2 \%$ in its measurement allows to probe values of $\Lambda$ slightly larger than $5 \mathrm{TeV}$. The forward-backward asymmetry as well as the $\alpha_{t}$ parameter are less sensitive to this choice, as shown in Fig. 27b where we have set $\delta f_{1}=\delta f_{3}=0$. Indeed, they are much more sensitive to the anomalous magnetic moments than to the change in the $f_{1}$ and $f_{3}$ form factors contrary to $R_{t}$. In Fig. $27 \mathrm{c}$ the same three observables are shown when the anomalous couplings to the photon are switched off (this happens for instance in dynamical symmetry breaking models where only the couplings to the $Z$ boson are expected to be non-universal). The deviations are much smaller in $R_{t}$ than in the previous case (this is due to the fact that the photon exchange dominates in the cross section) but they are much larger in $A_{t}$. Scales of the order of $2 \mathrm{TeV}$ can be probed in this case.

The possibility of turning the $e^{+} e^{-}$collider intto a $\gamma \gamma$ collider using backscattered laser beams, can be exploited to measure the $\gamma \bar{f} f$ couplings independently of the $Z \bar{f} f$ couplings. Assuming the total energy of the $\gamma \gamma$ collider to be $0.4 \mathrm{TeV}$, we show in Fig. 28 the deviations in the total cross section as a function of $\Lambda$ in the three cases: $\delta f_{1}=s / \Lambda^{2}, f_{2}=m_{t}^{2} / \Lambda^{2} ; \delta f_{1}=s / \Lambda^{2}, f_{2}=0$ and $\delta f_{1}=0, f_{2}=m_{t}^{2} / \Lambda^{2}$. As it can be seen, they are larger than in the $e^{+} e^{-}$case and 
the sensitivity to the anomalous magnetic moments is slightly better.

Figure 28: Deviations of the total cross section $\gamma \gamma \rightarrow t \bar{t}$ from the SM value for different values of the form-factors $f_{i}$; at $\sqrt{s}_{\gamma \gamma}$ is fixed to $400 \mathrm{GeV}$.

Hence, a $500 \mathrm{GeV}$ c.m. energy $e^{+} e^{-}$collider is a unique facility to probe the static properties of the top quark. A measurement of the total cross section in the $e^{+} e^{-}$or $\gamma \gamma$ modes with an accuracy of $2 \%$ allows to probe compositeness scale values up to $10 \mathrm{TeV}$. This corresponds to a radius of the order of $10^{-16} \mathrm{~cm}$. Furthermore, for light fermions the present limits can be greatly improved.

\section{3 $q \bar{q} \gamma \gamma$ Contact Interactions and Diphoton Production}

Instead of the direct production of new particles, physics beyond the SM may first appear as deviations in observables away from SM expectations, such as in the rates for rare processes or in precision electroweak tests. Another possibility is that deviations in cross sections of order unity may be observed once sufficiently high energy scales are probed. This kind of new physics can generally be parameterized via a finite set of non-renormalizable contact interactions, an approach which is quite popular in the literature 78]. In fact, limits already exist from a number of experiments on the scales associated with contact interactions of various types [79]. Here [80, we explore the capability of both the Tevatron and LHC to probe the existence of flavor-independent (apart from electric charge), $q \bar{q} \gamma \gamma$ contact interactions of dimension-8. Searches for such operators, with the quarks replaced by electrons, have already been performed at TRISTAN and LEP [81] and have resulted in a lower bound of approximately $140 \mathrm{GeV}$ on the associated mass scale.

To be definitive, we will follow the notation employed by 82 as well as by the ALEPH Collaboration [81] and assume that these new interactions are parity conserving. In this case we can parameterize the $q \bar{q} \gamma \gamma$ contact interaction as

$$
\mathcal{L}=2 i e^{2} Q_{q}^{2} \Lambda^{-4} F^{\mu \sigma} F_{\sigma}^{\nu} \bar{q} \gamma_{\mu} \partial_{\nu} q
$$

where $Q_{q}$ is the quark charge and $\Lambda$ is the associated mass scale. The most obvious manifestation of this new operator is to modify the conventional Born-level partonic $q \bar{q} \rightarrow \gamma \gamma$ differential cross section so that it now takes the form

$$
\frac{d \hat{\sigma}}{d z}=Q_{q}^{4} \frac{2 \pi \alpha^{2}}{3 \hat{s}}\left[\frac{1+z^{2}}{1-z^{2}} \pm 2 \frac{\hat{s}^{2}}{4 \Lambda_{ \pm}^{4}}\left(1+z^{2}\right)+\left(\frac{\hat{s}^{2}}{4 \Lambda_{ \pm}^{4}}\right)^{2}\left(1-z^{4}\right)\right]
$$

where $\hat{s}, z$ are the partonic c.m. energy and cosine of the c.m. scattering angle, $\theta^{*}$, respectively. Note that we have written $\Lambda_{ \pm}$in place of $\Lambda$ in the equation above to indicate that the limits we obtain below will depend upon whether the new operator constructively or destructively interferes with the SM contribution. 
There are two major effects due to finite $\Lambda$ : $(i)$ Clearly, once $\hat{s}$ becomes comparable to $\Lambda^{2}$, the parton-level differential cross section becomes less peaked in the forward and backward directions implying that the photon pair will generally be more central and will occur with higher average $p_{t}$ 's. (ii) When integrated over parton distributions the resulting cross section will lead to an increased rate for photon pairs with large $\gamma \gamma$ invariant masses. Thus we employ strict $\eta$ and $p_{t}$ cuts on both photons to reduce backgrounds from SM processes.

In presenting numerical results, we integrate the invariant diphoton mass distribution above a given fixed minimum value of the diphoton mass, $M_{\gamma \gamma}^{\min }$, subsequent to making all the other cuts. In order to get an estimate for the event rates involved, we scale this integrated cross section by a luminosity appropriate to the Tevatron or the LHC, i.e., $20 \mathrm{pb}^{-1}$ and $100 \mathrm{fb}^{-1}$, respectively. Figs. 29a-b compare the SM diphoton cross section as a function of $M_{\gamma \gamma}^{\min }$ with the constructive interference scenario for various values of the $\Lambda$ parameter.

Assuming that no event excesses are observed, we can ask for the limits that can be placed on $\Lambda_{ \pm}$as the Tevatron integrated luminosity is increased. To do this we perform a Monte Carlo study, first dividing the $M_{\gamma \gamma}^{\min }$ range above 100 $\mathrm{GeV}$ into nine steps of $50 \mathrm{GeV}$. Events are generated using the SM as input and are then fit to the resulting $\Lambda_{ \pm}$dependent fitting function. For a luminosity of $100(250,500,1000,2000) \mathrm{pb}^{-1}$ we obtain the bounds $\Lambda_{+}>487(535,575,622,671)$ $\mathrm{GeV}$ and $\Lambda_{-}>384(465,520,577,635) \mathrm{GeV}$, respectively, at $95 \%$ CL. Correspondingly, for a similar analysis at the LHC, we find with an integrated luminosity of $100 \mathrm{fb}^{-1}$, the $95 \% \mathrm{CL}$ bounds of $\Lambda_{+}>2.83 \mathrm{TeV}$ and $\Lambda_{-}>2.88 \mathrm{TeV}$.

Figure 29: (a) Diphoton pair event rate, scaled to a luminosity of $20 \mathrm{pb}^{-1}$, as a function of $M_{\gamma \gamma}^{\min }$ at the Tevatron subject to the cuts discussed in the text. The solid curve is the QCD prediction, while from top to bottom the dash dotted curves correspond to constructive interference with the SM and a compositeness scale associated with the $q \bar{q} \gamma \gamma$ operator of $\Lambda_{+}=0.2,0.3,0.4,0.5$, and $0.6 \mathrm{TeV}$ respectively. (b) Same as (a), but for the LHC scaling to a lumonosity of $100 \mathrm{fb}^{-1}$. From top to bottom the dash dotted curves now correspond to $\Lambda_{+}=0.75,1.0,1.25,1.5,1.75$ and $2.0 \mathrm{TeV}$ respectively.

\subsection{New resonant structures}

In this subsection we will discuss the indirect effects of Technicolour-like vector particles that are strongly coupled to the SM gauge bosons in the process $e^{+} e^{-} \rightarrow$ $\bar{f} f$. The high-precision LEP1 data have already set rather stringent bounds on Technicolour models [83], based on their effect to the quantity $S$ [83] a combination of the one-loop SM vector boson self-energies. Recently, a general formalism has been established 84 which allows to calculate the relevant one-loop self-energy corrections to processes at high energies $e^{+} e^{-}$colliders.

The main idea is that of expressing the various effects in the form of a oncesubtracted dispersion integral, and of fixing the necessary subtraction constants 
by suitable model-independent LEP1 results. In this way, one is led to a compact representation of several observables which presents two main advantages. The first one is that it allows to express new physics contributions through convergent integrals. The second one is that LEP1 constraints are automatically incorporated in the expressions of the observables. For example, the cross section for muon production at a c.m. energy $\sqrt{q^{2}}, \sigma_{\mu}\left(q^{2}\right)$, at the one-loop level takes the form

$$
\begin{aligned}
\sigma_{\mu}^{S E}\left(q^{2}\right)= & \frac{4 \pi q^{2}}{3}\left\{\left[\frac{\alpha\left(M_{Z}^{2}\right)}{q^{2}}\right]^{2}\left[1+2 D_{\gamma}\left(q^{2}\right)\right]+\frac{1}{\left(q^{2}-M_{Z}^{2}\right)^{2}+M_{Z}^{2} \Gamma_{Z}^{2}}\right. \\
& {\left.\left[\frac{3 \Gamma_{l}}{M_{Z}}\right]^{2}\left[1-2 D_{Z}\left(q^{2}\right)-\frac{16 s_{1}^{2} v_{1}}{1-v_{1}^{2}} D_{\gamma Z}\left(q^{2}\right)\right]\right\} }
\end{aligned}
$$

where $\Gamma_{l}$ is the leptonic $Z$ width, $\alpha\left(M_{Z}^{2}\right)=[1 \pm 0.001] / 128.87$ and

$$
\begin{aligned}
D_{\gamma}\left(q^{2}\right) & =-\left(q^{2}-M_{Z}^{2}\right) / \pi \mathcal{P} \int_{0}^{\infty} d s \operatorname{Im} F_{\gamma}(s)\left(s-q^{2}\right)^{-1}\left(s-M_{Z}^{2}\right)^{-2} \\
D_{Z}\left(q^{2}\right) & =\left(q^{2}-M_{Z}^{2}\right) / \pi \mathcal{P} \int_{0}^{\infty} d s s \operatorname{Im} F_{Z Z}(s)\left(s-q^{2}\right)^{-1}\left(s-M_{Z}^{2}\right)^{2} \\
D_{\gamma Z}\left(q^{2}\right) & =\left(q^{2}-M_{Z}^{2}\right) / \pi \mathcal{P} \int_{0}^{\infty} d s \operatorname{Im} F_{\kappa^{\prime}}(s)\left(s-q^{2}\right)^{-2}\left(s-M_{Z}^{2}\right)^{-1}
\end{aligned}
$$

with $F_{\kappa}^{\prime}=c_{1} / s_{1}, F_{Z \gamma}, s_{1}^{2} c_{1}^{2}=\pi \alpha /\left(\sqrt{2} G_{\mu} M_{Z}^{2}\right)$ and $s_{1}^{2}=1-c_{1}^{2} \simeq 0.217, v_{1}=1-4 s_{1}^{2}$. The imaginary parts which appear in these expressions are constructed from the self-energies; for Technicolour models, they are separately gauge-invariant. Similar representations can be established for several other observables like forwardbackward and polarization asymmetries, etc.. For each observable, one finally obtains an expression that include the full effect of the oblique correction at one-loop in the form: $O\left(q^{2}\right)=c_{0}\left[1+c_{\gamma} D_{\gamma}\left(q^{2}\right)+c_{Z} D_{Z}\left(q^{2}\right)+c_{\gamma Z} D_{\gamma Z}\left(q^{2}\right)\right]$, where the analytic expressions of the various coefficients can be found in [84].

One can use this formalism to calculate the possible effects of a pair of vector $(\mathrm{V})$ and axial vector $(\mathrm{A})$ resonances strongly coupled to the photon and to the $Z$ boson. The parameters which enter the expressions of the imaginary parts of the various spectral functions are the couplings $F_{V, A}$ and the masses $M_{V, A}$ (assumed to be larger than $\sqrt{q^{2}}$ ). Two different theoretical models have been considered:

(I) A Technicolour-like framework in which the validity of the two Weinberg sum rules 85] are exploited. Only their very general consequence, i.e. the positivity of $S$ are retained. In a zero-width approximation (in practice, one needs to use a finite width description of the $\mathrm{V}, \mathrm{A}$ resonances) one has: $S=4 \pi\left[F_{V}^{2} / M_{V}^{2}-\right.$ $\left.F_{A}^{2} / M_{A}^{2}\right]=4 \pi\left(F_{\pi}^{2} / M_{V}^{2}\right)\left[1+M_{V}^{2} / M_{A}^{2}\right]$. The present constraint on $S$ is $-0.9 \leq S \leq$ 0.4 [86]. In this model only the positive upper bound is effective.

(II) The constraints due to the Weinberg sum rules are released, a choice which has the consequence of introducing one more degree of freedom since it eliminates the theoretical relation between $F_{V}$ and $F_{A}$. As a consequence, $S$ can now take negative values, and in addition the strength of the ratios $F_{V} / M_{V}$ and $F_{A} / M_{A}$ is 
no more bounded. The limiting case of a strongly interacting regime for which the Figure 30.

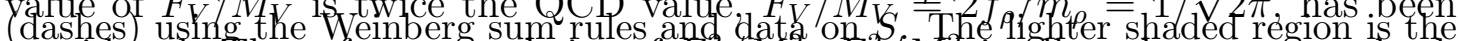

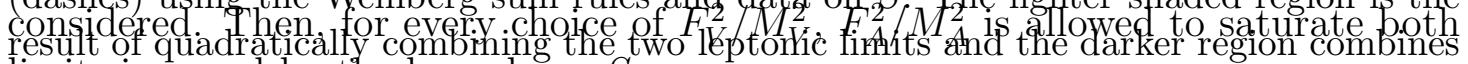
limits imposed thy the bounds on $S_{\text {and }}$ sospond to $M_{A}=(1.1,1.6) M_{V}$. (b) Limits obtained when released from the Weinberg sum rules but imposing the $F_{V} / M_{V}$ limitation from $\sigma_{\mu}$ (vertical,dotted), $A_{\tau}$ (vertical dashed), $A_{L R}$ (dotdashed), $R_{b, \mu}$ (shortdashed), $R^{(5)}$ (dotted), $A_{F B, \mu}$ (long dashed). The solid lines show $M_{A}=(1.0,1.6) M_{V}$.

Assuming a certain accuracy for the measurement of each observable, one accordingly obtains the observability limit of the self-energy effect that is translated in an upper bound on the masses $M_{V, A}$. For a $1 \mathrm{TeV} e^{+} e^{-}$collider the assumed accuracies are of a relative one percent for $\sigma_{\mu}, A_{F B, \mu}, A_{L R, h}, R^{(5)}$, two percent for $R_{b, \mu}$ and five percent for $A_{\tau}$. Results are shown in Fig. 30 for both models, the different curves corresponding to the various observables, and the shaded area to the combined overall mass bounds. In model (I) the resulting bounds on $M_{V, A}$ are located in the $2 \mathrm{TeV}$ range, and are rather strongly correlated; the only hadronic observable which contributes appreciably is $A_{L R, h}$ and allows to improve the pure leptonic result by about $200 \mathrm{GeV}$. In model (II) the effect of releasing the validity of the Weinberg sum rule is roughly that of increasing the bounds on $\left(M_{V}, M_{A}\right)$ from the $2 \mathrm{TeV}$ to the $4 \mathrm{TeV}$ region. Compared to the results obtained in 84, an improvement by a factor 6-8 as compared to the LEP2 case. The explored mass range of $M_{V} / M_{A}$ should be able to give a definite hint of the existence of Technicolour-like resonances or of any other strongly coupled vector boson. 


\section{References}

[1] For reviews on exotic fermions see, A. Djouadi, D. Schaile and C. Verzegnassi [conv.] et al., Proceedings of the Workshop " $e^{+} e^{-}$Collisions at $500 \mathrm{GeV}$ : the Physics Potential", Report DESY 92-123A+B, P. Zerwas, ed; P. Langacker and D. London, Phys. Rev. D38 (1988) 244; E. Nardi, E. Roulet and D. Tommasini, Nucl. Phys. B386 (1992) 239.

[2] M. Davier, Rapporteur talk at the Lepton-Photon Conference, Geneva 1991, Preprint LAL-91-48 (1991).

[3] For a review: J. Hewett and T. G. Rizzo, Phys.Rep. 183, 193 (1989).

[4] For a review: J. Maalampi and M. Roos, Phys. Rep. 186, 53 (1990).

[5] I. Montvay, Phys. Lett. B205, 315 (1988).

[6] R. Robinett and J. Rosner, Phys. Rev. D25, 3036 (1982); P. Langacker, R. W. Robinett and J. L. Rosner, Phys. Rev. D30, 1470 (1984); V. Barger et al., Phys. Lett. B118, 68 (1982).

[7] W. Buchmüller and C. Greub, Nucl. Phys. B363, 345 (1991).

[8] W. Buchmüller and C. Greub, Nucl. Phys. B381, 109 (1992).

[9] M. Drees, Nucl. Phys. B298, 333 (1988); W. Buchmüller, C. Greub and P. Minkowski, Phys. Lett. B267, 395 (1991).

[10] M. Chanowitz, M. Furman and I. Hinchliffe, Phys. Lett. B78, 285 (1978); F. Csikor and I. Montvay, Phys. Lett. B231, 503 (1990).

[11] M. Gronau, C.N. Lung and J. L. Rosner, Phys. Rev. D29 (1984) 2539; J. L. Rosner, Com. Nucl. Part. Phys. 15 (1986) 195; R. W. Robinett, Phys. Rev. D33 (1986) 1908; J. L. Rosner, Nucl. Phys. B248 (1984) 503; V. Barger et al., Phys. Rev. D33 (1986) 1912; T. G. Rizzo, Phys. Rev. D34 (1986) 1438; R. W. Robinett, Phys. Rev. D33 (1986) 1908; F. Gilman, Comm. Nucl. Part. Phys. 16 (1986) 231; K. Hagiwara and D. Zeppenfeld, Nucl. Phys. B274 (1986) 1; C. Ahn et al., SLAC-PUB-0329 (1988); F. del Aguila, E. Laermann and P. M. Zerwas, Nucl. Phys. B297 (1988) 1; M. Dittmar et al., Nucl. Phys. B332 (1990) 1; F. M. L. Almeida et al., Phys. Rev. D44 (1991) 2836; J. Maalampi, K. Mursula and R. Viopionperä, Nucl. Phys. B372 (1992) 23.

[12] For a recent discussion on exotic fermions properties, decays and production at $e^{+} e^{-}$colliders, and for references on previous work, see A. Djouadi, Z. Phys. C63, 317 (1994) and G. Azuelos and A. Djouadi, Z. Phys. C63, 327 (1994). 
[13] For reviews on substructure in the fermionic sector, see: M. Peskin, Proc. of the 10th Int. Symp. on Lepton-Photon Interactions at High-Energies, Bonn FRG, August 1981; H. Terazawa, Proc. of the Europhysics Topical Conference on Flavor Mixing in Weak Interactions, Erice Italy, March 1984; W. Buchmüller, Lectures given at the 24th Int. Wochenuniversität für Kernphysik, Schladmig Austria, Feb. 1985; R. Peccei, Proc. of the Lake Louise Winter Institute, Lake Louise Canada, Feb. 1987; F. Boudjema, Int. J. Mod. Phys. A6 (1991) 1.

[14] F. Low, Phys. Rev. Lett. 14, 238 (1965); N. Cabibbo, L. Maiani and Y. Srivastava, Phys. Lett. B139, 459 (1984); F. M. Renard, Phys. Lett. B116, 269 (1982) 264; A. DeRujula, L. Maiani and R. Petronzio, Phys. Lett. B140, 253 (1984); J. Kühn, H.D. Tholl and P.M. Zerwas, Phys. Lett. B158, 270 (1985); K. Hagiwara, S. Komamyia and D. Zeppenfeld, Z. Phys. C29,115 (1985); F. Boudjema and A. Djouadi, Phys. Lett. B240, 485 (1990); I. F. Ginzburg and D. Yu. Ivanov, Phys. Lett. B276 (1992) 218; E. Boos, A. Belyaev and A. Phukov, Phys. Lett. B296 (1992) 452.

[15] For a detailed analysis on excited fermions and a complete set of references on previous work, see F. Boudjema, A. Djouadi and J. L. Kneur, Z. Phys. C57, 425 (1992).

[16] C. Bétourné et al., Phys. Lett. 17 (1965) 70; H.J. Behrend et al., Phys. Rev. Lett. 15 (1965) 900.

[17] For a review, see W. Buchmüller, talk presented at the First Workshop on Linear Colliders, Saariselkä, Finland, September 1991.

[18] I. Bigi, G. Köpp and P. M. Zerwas, Phys. Lett. 166B, (1986), 238; A. Djouadi et al., Z. Phys. C46 (1990), 679.

[19] W. Buchmüller, R. Rückl, and D. Wyler, Phys. Lett. B191, 442 (1987); W. Buchmüller, and D. Wyler, Phys. Lett. B177, 377 (1986).

[20] P. H. Frampton, Mod. Phys. Lett. A7 (1992) 559.

[21] P. H. Frampton and D. Ng, Phys. Rev. D45, 4240 (1992).

[22] E. Nardi, E. Roulet, D. Tommasini, contribution to this report and CERNTH-7443-94.

[23] P. Langacker and S. Uma Sankar, Phys. Rev. D40 (1989) 1569.

[24] T. G. Rizzo, Phys. Rev. D38, 2076 (1986); F. Csikor and Z. Fodor, Phys. Lett. B287, 358 (1992). 
[25] A. Adriani et al., L3 Collaboration., Phys. Lett. B295, 371 (1992).

[26] M. Sher, contribution to this report and in preparation.

[27] Z. Berezhiani and E. Nardi, HEP-PH-9411249.

[28] P. Frampton and T. Kephart, Phys. Rev. D47, 3655 (1993); K. Griest and M. Sher, Phys. Rev. D42, 3834 (1990) and Phys. Rev. Lett. 64, 135 (1990).

[29] D. Ng, J. Ng, R. Garisto and J. Soares, contribution to this report.

[30] G. Azuelos, contribution to this report.

[31] C. Gonzalez-Garcia et al., Phys. Lett. B280, 313 (1992).

[32] For a review of the LRM and original references, see R.N. Mohapatra, Unification and Supersymmetry, (Springer, New York, 1986). See also, T.G. Rizzo, SLAC-PUB-95-6741.

[33] T.G. Rizzo, Phys. Lett. B116, 23 (1982); D. London, G. Belanger, and J.N. Ng, Phys. Lett. B188, 155 (1987); J. Maalampi, A. Pietilä, and J. Vuori, Phys. Lett. B297, 327 (1992) and Turku University report FL-R9 (1992); M.P. Worah, Enrico Fermi Institute report EFI 92-65 (1992); see also T.G. Rizzo in, Proceedings of the Workshop on Physics and Experiments with Linear $e^{+} e^{-}$Colliders, Waikoloa, Hawaii, April 1993, edited by F.A. Harris et al., (World Scientific, Singapore, 1993).

[34] T.G. Rizzo, Phys. Rev. D50, 5602 (1994).

[35] K. Huitu, J. Maalampi and M. Raidal, contribution to this report, and Phys. Lett. B328, 60 (1994) and Nucl. Phys. B420, 449 (1994); see also J. Gluza and M. Zralek, University of Silesia report SU-TP-1/95, 1995.

[36] C.A. Heusch and P. Minkowski, contribution to this report; for original references, see C.A. Heusch, SCIPP 94/24 (1994), to be published in Nucl. Phys. B; C.A. Heusch, P. Minkowski, BUTP 95/11, SCIPP 95/07, to be published; C.A. Heusch, P. Minkowski, Nucl. Phys. B416, 3 (1994).

[37] F. Cuypers et al., Phys. Lett. B325, 243 (1994).

[38] V. Barger et al., Phys. Rev. D50, 6704 (1994).

[39] M. Spira, contribution to this report.

[40] C. Greub, contribution to this report.

[41] G. Bhattacharya, P. Kalyniak and K. Peterson, contribution to this report. 
[42] See e.g., R. K. Ellis and J.C. Sexton, Nucl. Phys. B282, 642 (1987)

[43] P. Nason, S. Dawson and R.K. Ellis, Nucl. Phys. B303, 607 (1988); W. Beenakker et. al., Phys. Rev. D40, 54 (1989); Nucl. Phys. B351, 507 (1991)

[44] V. Barger and R.J.N. Phillips, Phys. Rev. Lett. 55, 2752 (1985); H. Baer, V. Barger, H. Goldberg and R.J.N. Phillips, Phys. Rev. D37, 3152 (1988)

[45] P.N. Harriman, A.D. Martin, R.G. Roberts and W.J. Stirling, Phys. Rev. D42, 798 (1990)

[46] J. Kühn and P.M. Zerwas, Phys. Lett. B147, 189 (1984).

[47] P. Mery et al., Z. Phys. C46 (1990) 229.

[48] F. M. Renard, Z. Phys. C14, 209 (1982).

[49] J. L. Kneur, contribution to this report.

[50] U. Baur, M. Spira, P.M. Zerwas, Phys. Rev. D42, 815 (1990). U. Baur, I. Hinchliffe and D. Zeppenfeld, Proceedings, Workshop From Colliders to Supercolliders, Madison (Wisconsin) 1987.

[51] G. Bélanger, D. London and H. Nadeau, contribution to this report. For details, see Phys. Rev. D49, 3140 (1994), and references therein, by these authors.

[52] J.L. Hewett and S. Pakvasa, Phys. Lett. B227, 178 (1989).

[53] M.A. Doncheski and S. Godfrey, Phys. Rev. D49, 6220 (1994). See also O.J. Éboli et al., Phys. Lett. B311, 147 (1993).

[54] M. Glück, E. Reya and A. Vogt, Phys. Rev. D46, 1973 (1992).

[55] J.L. Hewett and S. Pakvasa, Phys. Lett. B227, 178 (1989); H. Nadeau and D. London, Phys. Rev. D47, 3742 (1993); G. Bélanger, D. London and H. Nadeau, in Ref. [51]; J. E. Cieza Montalvo and O.J.P. Éboli, Phys. Rev. D47, 837 (1993); O.J. Éboli, E.M. Gregores, M.B. Magro, P.G. Mercadante, and S.F. Novaes, Phys. Lett. B311, 147 (1993); M.A. Doncheski and S. Godfrey, Phys. Rev. D49, 6220 (1994); T.M. Aliev and Kh.A. Mustafaev, Yad. Fiz. 58, 771 (1991).

[56] M.A. Doncheski and S. Godfrey, Ottawa-Carleton report OCIP/C 94-7, 1994.

[57] J. A. Hassan and D. J. Pilling, Nucl. Phys. 187, 563 (1981); Z. Xu, Phys. Rev. D30, 1440 (1984); A. Nicolaidis, Nucl. Phys. B163, 156 (1980). 
[58] J. Blümlein, contributed to this report; see also J. Blümlein and E. Boos, Nucl. Phys. (Proc. Suppl.), 37B, 181, (1994), and references therein.

[59] J.L. Hewett and T.G. Rizzo, Phys. Rev. D36, 3367 (1987); H. Dreiner et al., Mod. Phys. Lett. A3, 443 (1988). This analysis was updated for this report by J.L. Hewett.

[60] J.L. Hewett, T.G. Rizzo, S. Pakvasa, A. Pomarol, and H.E. Haber, contributed to this report. See also, the report of these authors in the Proceedings of the Workshop on Physics at Current Accelerators and Supercolliders, Argonne National Laboratory, June 2-5, 1993 .

[61] J.L. Hewett and S. Pakvasa, Phys. Rev. D37, 3165 (1988).

[62] K. Hagiwara et al., Nucl. Phys. B282, 253 (1987).

[63] E. Ma, Phys. Rev. D36, 274 (1987) and Mod. Phys. Lett. A3, 319 (1988); K.S. Babu et al., Phys. Rev. D36, 878 (1987); V. Barger and K. Whisnant, Int. J. Mod. Phys. A3, 879 (1988); J.F. Gunion et al., Int. J. Mod. Phys. A2, 118 (1987); T.G. Rizzo, Phys. Lett. B206, 133 (1988).

[64] P. Chiappetta for this report.

[65] N. Leporé, B. Thorndyke, H. Nadeau and D. London, contribution to this report. For details of this analysis, Phys. Rev. D50 (1994) 2031 by these authors.

[66] P.H. Frampton et al., Phys. Lett. B317, 369 (1993); J. Agrawal, P.H. Frampton, and D. Ng, Nucl. Phys. B386, 267 (1992); T.G. Rizzo, Phys. Rev. D45, 42 (1992) and Phys. Rev. D46, 910 (1992).

[67] F. Abe et al., CDF Coll., Phys. Rev. Lett. 73, 225 (1994), Phys. Rev. D50, 2966 (1994), and Fermilab reports Fermilab-PUB-94/411-E, 1994 and PUB95/022-E, 1995; S. Abachi et al., D0 Collaboration, Phys. Rev. Lett. 72, 2138 (1994)and Fermilab reports Fermilab-PUB-94/354-E, 1994 and PUB-95/028E, 1995. CDF and D0 Collaboration presentations at Fermilab, March 2, 1995.

[68] D. Schaile, talk presented at, and LEP (CERN) reports LEPEWWG/94-02, LEPTAU /94-02, LEPLINE/94-01, and LEPHF/94-03, submitted to the $27^{\text {th }}$ International Conference on High Energy Physics, Glasgow, Scotland, July 20-27, 1994.

[69] G. Kane, G.A. Ladinsky, and C.P. Yuan, Phys. Rev. D45, 124 (1992); C.P. Yuan, Phys. Rev. D45, 782 (1992); D. Atwood, A. Aeppli, and A. Soni, Phys. Rev. Lett. 69, 2754 (1992); M. Peskin, talk presented at the Second International Workshop on Physics and Experiments at Linear $e^{+} e^{-}$Collider, 
Waikoloa, HI, April 1993; M. Peskin and C.R. Schmidt, talk presented at the First Workshop on Linear Colliders, Saariselkä, Finland, September 1991; P. Zerwas, ibid.; W. Bernreuther et al., in Proceedings of the Workshop on $e^{+} e^{-}$ Collisions at $500 \mathrm{GeV}$, The Physics Potential, (DESY, Hamburg) ed. by P. Igo-Kemenes and J.H. Kühn, 1992; A. Djouadi, ENSLAPP-A-365-92 (1992); D.O. Carlson, E. Malkawi, and C.-P. Yuan, Michigan State report MSUHEP94/05, 1994; J.L. Hewett and T.G. Rizzo, Phys. Rev. D49, 319 (1994).

[70] A. Kagan, talk presented at the Fourth International Conference on Physics Beyond the Standard Model, Lake Tahoe, CA, December 13-18, 1994 and references therein.

[71] E. Laenen, J. Smith, and W.L. van Neerven, Phys. Lett. B321, 254 (1994); A.D. Martin, W.J. Stirling and R.G. Roberts, Phys. Rev. D50, 6734 (1994).

[72] D. Atwood, A. Kagan and T. Rizzo, SLAC-PUB-6580, 1994; T.G. Rizzo, Phys. Rev. D50, 4478 (1994); T.G. Rizzo, SLAC-PUB-95-6758, 1995.

[73] For a review see M. E. Peskin, Proc. Int. Symp. on Lepton and Photon Interactions at High Energies, Kyoto 1985, eds. M. Kunoma and K. Takahashi.

[74] For a compilation see F. Boudjema, F. M. Renard [conv.] et al. in $Z$ Physics at LEP, eds. G. Altarelli, R. Kleiss and C. Verzegnassi, CERN 89-08.

[75] A. Djouadi and M. Spira, Phys. Lett. B228, (1989) 443.

[76] R. D. Peccei and X. Zhang, Nucl. Phys. B337, (1990) 269.

[77] A. Djouadi, Proceedings of the Workshop " $e^{+} e^{-}$collisions at $500 \mathrm{GeV}$ : the Physics Potential", Report DESY 92-123B, P. Zerwas (ed), p. 831.

[78] See, for example, E. Eichten, K. Lane and M. Peskin, Phys. Rev. Lett. 50, 811 (1983); B. Schrempp, F. Schrempp, N. Wermes and D. Zeppenfeld, Nucl. Phys. B296, 1 (1988).

[79] K. Hikasa et al., Particle Data Group, Phys. Rev. D45, II (1992).

[80] T.G. Rizzo, SLAC-PUB-6579, to appear in Phys. Rev. D51, (1995).

[81] D. Buskulic et al., ALEPH Collaboration, Z. Phys. C59, 215 (1993); P. Abreu et al., DELPHI Collaboration, Phys. Lett. B268, 296 (1991); O. Adriani et al., L3 Collaboration, Phys. Lett. B288, 404 (1992); M.Z. Akrawy et al., OPAL Collaboration, Phys. Lett. B257, 531 (1991); H.J. Kim et al., AMY Collaboration, KEK report 89-52, 1992; K. Shimozawa et al., TOPAZ Collaboration, Phys. Lett. B284, 144 (1992); K. Abe et al., VENUS Collaboration, Z. Phys. C45, 175 (1989). 
[82] See, for example, P. Méry, M. Perrottet and F.M. Renard, Z. Phys. C38, 579 (1988).

[83] M.E. Peskin and T. Takeuchi, Phys. Rev. D46, (1991) 381.

[84] J. Layssac, F.M. Renard and C. Verzegnassi, Phys. Rev. D48, (1993) 4037, D49, (1994) R2143 and D49, (1994) 3650.

[85] S. Weinberg, Phys. Rev. Lett. 18, (1967) 507.

[86] We use the values for $\epsilon_{3}$ given by G. Altarelli, CERN-TH-7319/94.

[87] S. Weinberg, Phys. Rev. D13, (1976) 974 and D19, (1979) 1277; L. Susskind, Phys. Rev D20, (1979) 2619; E. Fahri and L. Susskind, Phys. Rev D20, (1979) 3404. 


\begin{tabular}{|c||c|c|c|}
\hline Process & $E^{ \pm} e^{\mp}$ & $e^{+} e^{-} Z$ & $Z Z$ \\
\hline$\sigma[\mathrm{fb}]$ & 9.5 & 4960 & 615 \\
\hline$\times$ B.R. & 2.19 & 3470 & 28.8 \\
\hline one $e^{+} e^{-}$pair & 1.74 & 93.0 & 23.0 \\
$330<M_{E}<370$ & 1.56 & 11.7 & 5.30 \\
$85<M_{Z}<105$ & 1.41 & 5.84 & 2.87 \\
$\left|M_{l l}-M_{Z}\right|>12$ & 1.39 & 5.18 & 1.02 \\
$\cos \theta_{l l}<0.5$ & 1.33 & 4.32 & 0.56 \\
$f\left(M_{E}, \cos \theta_{Z}\right)$ & 1.30 & 1.90 & 0.43 \\
kinem. cuts & 1.30 & 1.55 & 0.39 \\
\hline
\end{tabular}

\begin{tabular}{|c||c|c|c|}
\hline Process & $N \nu$ & $e \nu W$ & $W W$ \\
\hline$\sigma[\mathrm{fb}]$ & 490 & 8610 & 2600 \\
\hline$\times$ B.R. & 13.7 & 5823 & 1140 \\
\hline one $e$ & 13.2 & 198 & 883 \\
$330<M_{N}<370$ & 12.5 & 11.9 & 100 \\
$70<M_{W}<90$ & 12.3 & 10.3 & 70.3 \\
$M_{l \nu}>120$ & 11.8 & 10.0 & 7.93 \\
$\cos \theta_{l \nu}<0.5$ & 11.7 & 10.0 & 7.80 \\
$f\left(M_{N}, \cos \theta_{Z}\right)$ & 11.7 & 10.0 & 7.80 \\
kinem. cuts & 11.7 & 10.0 & 4.13 \\
\hline
\end{tabular}

